\title{
STABILITY OF 2D FDTD ALGORITHMS WITH LOCAL MESH REFINEMENT FOR MAXWELL'S EQUATIONS*
}

\author{
A. R. ZAKHARIAN ${ }^{\dagger}$, M. BRIO ${ }^{\ddagger}$, C. DINEEN ${ }^{\S}$, AND J. V. MOLONEY
}

\begin{abstract}
We perform stability analysis on the finite-difference time domain method (FDTD) when extended to incorporate local space-time adaptive mesh refinement (AMR). The neutrally stable Yee algorithm becomes extremely sensitive to perturbations introduced by the interpolation schemes employed at grid refinement interfaces. In this paper we investigate the stability of a range of interpolation schemes using Gustafsson-Kreiss-Sundstrom-Trefethen (GKS-T) mode and reflection/transmission coefficients analysis on the infinite domain with a single interface. This analysis allows detection of trapping instabilities, exponentially growing modes, mode resonances with the interface and mode-mode resonances. We also apply matrix stability analysis for more complicated computational domains containing multiple grid refinement interfaces.
\end{abstract}

Key words. Maxwells's equations, FDTD, subgridding, local grid refinement, stability analysis

AMS subject classifications. 65M06, 65M50, 78M20

\section{Introduction}

Current technological advances illuminate the critical need for accurate Maxwell solvers beyond the standard Yee scheme capabilities [27]. In the last two decades there have been numerous investigations of local grid refinement strategies $[1,3,6,5,26$, $15,22]$. The main advantages of such methods are reduced computational resource requirements and increased computational efficiency over methods using uniform mesh size when the region of interest occupies a small portion of the domain. The effective application of AMR produces results comparable to that of using the finest mesh uniformly over the whole domain. AMR algorithms are designed to resolve fine geometrical details and/or resolve singularities in the solution. The trade off is additional code for grid management, more complicated data structures for grid representation and a need for interface boundary conditions. The adaptive mesh refinement algorithm, originally developed by Berger et. al. [3] for systems of hyperbolic conservation laws, has been successfully applied to applications in fluid dynamics, magnetohydrodynamics and astrophysics [13].

In AMR FDTD algorithms the original Yee scheme is applied throughout the domain with the exception of the grid interfaces. At grid interfaces various interpolation schemes may be employed to provide the missing boundary data for nested refinement domains. The motivation for a particular interpolation scheme is often of physical origin aiming at enforcing one or more constraints such as conservation of a discrete energy or divergence-free fields. The Yee FDTD algorithm by itself is second order accurate and neutrally stable. Deviation from these properties may only come about due to the interaction of the inner scheme with the interface interpolation algorithm. Therefore, the prime objective of this paper is the development of a grid interface interpolation algorithm that preserves the second-order accuracy of

\footnotetext{
${ }^{*}$ Received: November 29, 2005; accepted (in revised version): March 22, 2006. Communicated by Shi Jin.

${ }^{\dagger}$ College of Optical Sciences, the University of Arizona, Tucson AZ 85721, USA (armis@email.arizo na.edu).

$\ddagger$ Department of Mathematics at the University of Arizona, Tucson AZ 85721, USA.

$\S$ Department of Mathematics and ACMS at the University of Arizona, Tucson AZ 85721, USA.

TCollege of Optical Sciences, the University of Arizona, Tucson AZ 85721, USA.
} 
the original Yee scheme and is stable for long time integration. We would like to emphasize that we are interested here in long time stability in contrast to classical (Lax) stability for vanishing discretization parameters. For example, if the reflection coefficient at the interface is $R=1+O(\Delta t)^{2}$ as $\Delta t \rightarrow 0$, convergence is not prohibited according to Lax convergence theory. In practice, however, if $R>1$ instability may result for fixed, but small $\Delta t$ when the number of iterations is sufficiently large. The mode with Nyquist wavenumber is often the fastest growing mode is such a situation.

The Yee algorithm is neutrally stable, due to staggering in space and time, however, this symmetry is easily broken by the space-time interpolations required to produce the boundary conditions for the refined patches. At present there are no neutrally stable AMR FDTD algorithms, nor is it known if such a construction is even possible. The rigorous mathematical analysis of the stability of the AMR FDTD algorithms in the presence of the boundaries, interfaces and sources relies on Gustafsson-Kreiss-Sundstrom, and Trefethen's seminal contributions [9, 23, 24, 25]. The GKS theory investigates the stability of discrete initial boundary value problems using the Laplace-Fourier transform in time and space, respectively. It provides sufficient stability conditions only in certain one dimensional cases, but allows one to detect exponentially growing and possibly resonant mode instabilities. The GKS definition of "generalized" stability that allows for exponentially growing numerical solutions in time, with constant growth rate, is not applicable to the long time integration of Maxwell's equations in physical regimes containing no temporal growth. Trefethen's work has contributed a physical interpretation of the GKS theory in terms of boundary resonances and associates the trapping instability with a reflection coefficient being larger than unity. Combining both theories together leads to a series of tests to detect trapping instabilities, growing modes, mode-mode and mode-boundary resonances.

In [2] an AMR algorithm for a scalar advection equation in one space dimension was investigated for GKS-type instability without investigating for Trefethen's trapping instability. In this paper, it was pointed out that in contrast to the continuous case, the discrete solution should take into account that a single wave on the coarse grid generates two waves on the fine grid. In multidimensional applications GKS-Trefethen theory was applied by Higdon to a multidimensional wave equation $[10,11]$ in order to study modes that manifest themselves by an infinite reflection coefficient. An algorithm to detect possible GKS-unstable modes that require further investigation for systems in several space dimensions was presented in [21].

The stability of AMR algorithms in previously published papers is addressed using numerical tests rather than theoretical stability analysis. For example, [22, 16, 26] discuss 3D AMR FDTD algorithms. For semi-discrete approximation with continuous time and discrete space, $[22,15]$, showed that it is possible to produce an interpolation algorithm such that the resulting ODE preserves the symplectic structure of the original Maxwell method, and thus conserves the energy. No such construction is known for the fully discrete case.

Here we present a series of stability tests that involve reflection and transmission and a mode analysis of the solution on the infinite domain with a single interface and matrix stability analysis on finite domains. The analysis allows detection of trapping instabilities, exponentially growing modes, mode resonances with the interface and mode-mode resonances.

This paper is organized as follows. In section one, we briefly overview the basic properties of the Yee algorithm including discrete energy conservation. AMR algo- 
rithms for 2D Maxwell equations, describing both (TM) and (TE) mode propagation are presented in the next section. In section three we perform trapping and interface stability tests by computing reflection/transmission coefficients as well as mode analysis of the infinite domain with a single grid interface, and matrix stability analysis on finite domains with one refinement level. In section four, we present numerical examples that produce various trapping and interface instabilities for two dimensional domains.

\section{The Yee scheme dispersion relation}

Consider non-dimensional linear vector Maxwell's equations in isotropic, homogeneous non-dispersive media. For transverse magnetic (TM) mode propagation (ignorable z-coordinate) the equations are:

$$
\begin{aligned}
& \frac{\partial H_{x}}{\partial t}=-\frac{\partial E_{z}}{\partial y}, \\
& \frac{\partial H_{y}}{\partial t}=\frac{\partial E_{z}}{\partial x}, \\
& \frac{\partial E_{z}}{\partial t}=\frac{\partial H_{y}}{\partial x}-\frac{\partial H_{x}}{\partial y} .
\end{aligned}
$$

The (TE) mode propagation equations may be obtained by swapping electric and magnetic field variables. Numerical discretization on each level of refinement is done using the standard Yee scheme that is staggered in space and time and applied on a uniform mesh,

$$
\begin{array}{r}
H_{x i+1 / 2, j}^{n+1 / 2}-H_{x i+1 / 2, j}^{n-1 / 2}=-\nu_{y}\left(E_{z i+1 / 2, j+1 / 2}^{n}-E_{z i+1 / 2, j-1 / 2}^{n}\right), \\
H_{y i, j+1 / 2}^{n+1 / 2}-H_{y i, j+1 / 2}^{n-1 / 2}=\nu_{x}\left(E_{z i+1 / 2, j+1 / 2}^{n}-E_{z i-1 / 2, j+1 / 2}^{n}\right), \\
E_{z i+1 / 2, j+1 / 2}^{n+1}-E_{z i+1 / 2, j+1 / 2}^{n}=\nu_{x}\left(H_{y i+1, j+1 / 2}^{n+1 / 2}-H_{y i, j+1 / 2}^{n+1 / 2}\right) \\
-\nu_{y}\left(H_{x i+1 / 2, j+1}^{n+1 / 2}-H_{x i+1 / 2, j}^{n+1 / 2}\right),
\end{array}
$$

where the Courant numbers $\nu_{x}$ and $\nu_{y}$ are defined as $\Delta t / \Delta x$ and $\Delta t / \Delta y$, respectively.

In one space dimension, (dropping the $y$-dependence and $H_{x}$ component of the magnetic field), the numerical dispersion relation of the Yee scheme is

$$
\sin \left(\frac{\omega \Delta t}{2}\right)= \pm \nu \sin \left(\frac{k \Delta x}{2}\right),
$$

where $\Delta t$ and $\Delta x$ describe the grid size on the level of refinement considered. For an initial value problem the wave number $k$ is given and the frequency $\omega(k)$ is determined by the dispersion relation. The stability, defined as uniform boundedness of the numerical solution with respect to number of iterations, initial condition and the grid size, implies that $\nu<1$ [20]. The Yee algorithm is dispersive and non-dissipative since $\omega(k)$ is real function for real wavenumber $k$. The phase velocity $\omega / k$ and group velocity $\omega^{\prime}(k)$ approximate the exact dispersion relation $\omega= \pm k$ to second order accuracy near $k=0[20]$.

On the other hand, for a boundary value problem or a problem with a forcing term, the frequency $\omega$ is given or obtained by solving an eigenvalue problem and 
the corresponding wavenumber $k(\omega)$ is determined from the dispersion relation. The wavenumber $k$ becomes complex for values of $\omega$ larger than the cutoff frequency $\omega_{c}$, $\sin \left(\frac{\omega_{c} \Delta t}{2}\right)= \pm \nu$, obtained by substituting the Nyquist wavenumber $(k \Delta x=\pi)$ into the dispersion relation.

On the fine grid the dispersion relation normalized by the coarse space and time grid spacing is

$$
\sin \left(\frac{\omega \Delta t}{4}\right)= \pm \nu \sin \left(\frac{k \Delta x}{4}\right) .
$$

The dispersion relation (2.4) implies that in terms of the coarse grid, the fine cutoff frequency is double that on the coarse grid, if the grid size ratio is 1:2. Therefore on the fine grid the relevant frequencies, up to the fine cutoff frequency, include both propagating and non-propagating modes of the coarse grid. Another important observation for the forthcoming discussion is that two indistinguishable frequencies on the coarse grid, $\omega \Delta t$ and $\tilde{\omega} \Delta t=\omega \Delta t+2 \pi$, correspond to two distinct wavenumbers on the fine grid, $k$ defined by equation (2.4) and $\tilde{k}$ defined by the modified dispersion relation,

$$
\sin \left(\frac{\omega \Delta t+2 \pi}{4}\right)= \pm \nu \sin \left(\frac{\tilde{k} \Delta x}{4}\right)
$$

In the two dimensional case the Yee dispersion relation is as follows,

$$
\sin ^{2}\left(\frac{\omega \Delta t}{2}\right)=\nu^{2}\left[\sin ^{2}\left(\frac{k_{x} \Delta x}{2}\right)+\sin ^{2}\left(\frac{k_{y} \Delta y}{2}\right)\right] .
$$

For given real $k_{y} \Delta y$ and real $\omega \Delta t, k_{x} \Delta x$ is real only in the range

$$
0 \leq \sin ^{2}\left(\frac{k_{x} \Delta x}{2}\right) \leq 1
$$

or equivalently,

$$
\nu^{2} \sin ^{2}\left(\frac{k_{y} \Delta y}{2}\right) \leq \sin ^{2}\left(\frac{\omega \Delta t}{2}\right) \leq \nu^{2}+\nu^{2} \sin ^{2}\left(\frac{k_{y} \Delta y}{2}\right) .
$$

Total internal reflection results when the plane wave becomes non-propagating in $x$-direction, and is defined by the relation

$$
\sin ^{2}\left(\frac{\omega \Delta t}{2}\right)<\nu^{2} \sin ^{2}\left(\frac{k_{y} \Delta y}{2}\right) .
$$

Note that the highest frequency for total internal reflection corresponds to the Nyquist transverse wavenumber, $k_{y} \Delta y=\pi$, and is determined by the same relation as the cutoff frequency in the one dimensional case, $\sin (\omega \Delta t / 2)= \pm \nu$. Similarly to the one dimensional case, a single wave on the coarse grid in two dimensions will generate four waves on the fine grid, obtained by substituting $\omega$ by $\omega+2 \pi$ and $k_{y}$ by $k_{y}+2 \pi$ into the dispersion relation on the fine grid side,

$$
\sin ^{2}\left(\frac{\omega \Delta t+2 \pi p}{4}\right)=\nu^{2}\left[\sin ^{2}\left(\frac{k_{x}^{p, q} \Delta x}{4}\right)+\sin ^{2}\left(\frac{k_{y} \Delta y+2 \pi q}{4}\right)\right], \quad p, q=0,1 .
$$


For a given grid frequency $\omega \Delta t$ and transverse wavenumber $k_{y} \Delta y$, this dispersion relation determines four fine wavenumbers normal to the interface, $k_{x}^{p, q}, p, q=0,1$. This observation will be used in a later section to obtain exact analytical solutions of the reflection/transmission problem as well as mode amplitudes on an infinite domain with a single coarse-fine grid interface. The sign of the real and imaginary parts of the wavenumber $k_{x}^{p, q}$ are chosen as follows. For the propagating mode, $k_{x}^{p, q}$ is real and the sign is chosen so that the wave travels away from the interface. If the mode is evanescent, $k_{x}^{p, q}$ is complex, and the sign of the imaginary part of $k_{x}^{p, q}$ is chosen so that the mode decays to zero away from the interface.

2.1. Discrete energies for Yee algorithm. Consider a one dimensional Maxwell wave equation on a domain with periodic or Dirichlet boundary conditions:

$$
\frac{\partial H}{\partial t}=\frac{\partial E}{\partial x}, \frac{\partial E}{\partial t}=\frac{\partial H}{\partial x} .
$$

The energy defined as $\mathcal{E}(t)=\frac{1}{2} \int\left(|E|^{2}+|H|^{2}\right) d x$ is conserved. In the discrete case the following approximations were introduced in $[6,17,22]$,

$$
\begin{gathered}
\mathcal{E}_{1}(n)=\frac{1}{2} \sum_{j}\left[\left(E_{j}^{n}\right)^{2}+H_{j+1 / 2}^{n+1 / 2} H_{j+1 / 2}^{n-1 / 2}\right], \\
\mathcal{E}_{2}(n)=\frac{1}{2} \sum_{j}\left[\left(E_{j}^{n}\right)^{2}+\left(H_{j+1 / 2}^{n+1 / 2}\right)^{2}\right] .
\end{gathered}
$$

The first expression is a true system invariant valid for an arbitrary Courant number even in unstable situations when the magnetic part of $\mathcal{E}_{1}(n)$ becomes negative. Therefore, its conservation cannot guarantee that the high frequency modes will not be amplified. The second expression is positive and if it can be established that it remains uniformly bounded the stability of the algorithm will follow [17, 7]. When the method is convergent, $\mathcal{E}_{2}(n)$ is only an approximate invariant as its value oscillates around $\mathcal{E}_{2}(0)[18,17,22,29]$.

\section{Design of AMR FDTD algorithms}

A key step in AMR FDTD algorithm design is the construction of interface boundary conditions preserving the accuracy and stability properties of the original Yee algorithm. In our discussion we only consider a coarse-fine refinement ratio of 1:2 and treat the electric field as a primary variable.

The FDTD AMR algorithm consists of the following steps that are applied recursively at each refinement level:

(1) update coarse field values;

(2) provide ghost boundary values using space-time interpolation of nearby coarse and fine values;

(3) update fine field values;

(4) repeat steps 2 and 3 for the second update on the fine grid;

(5) update coarse $E$ fields parallel and adjacent to fine interfaces using newly updated fine $H$ fields.

Note that the ghost boundary conditions have to be provided for each time refinement level. A detailed pseudo-code of the (TM) algorithm is provided in [29] and the table of interpolation schemes used in each algorithm is given at the end of this section. 
Unlike algorithms with overlapping grids [4], where different levels of refinement are updated independently, each fine grid patch in our nested grid construction overlaps with the coarser grid region by one fine (ghost) grid computational cell.

In the context of material interfaces, second- and higher-order accuarate embedded FDTD methods ([31] and references therein) can be combined with our gridrefinement approach to account for the proper treatment of the material interfaces. Local modification of the update stencil can be applied on any given level of refinement, as long as it is away from grid refinement interfaces.

3.1. One dimensional interpolation algorithms. The one dimensional case requires spatial interpolation normal to the interface, to provide the ghost value at time level $n$, and an update to advance the ghost value to time level $n+1 / 2$. In the direction normal to the interface we applied linear, quadratic or cubic interpolations, abbreviated as Lin, Qu and Cu respectively,

$$
\begin{aligned}
& e_{z i-1 / 4}^{n}=\frac{2}{3} E_{z i-1 / 2}^{n}+\frac{1}{3} e_{z i+1 / 4}^{n}, \\
& e_{z i-1 / 4}^{n}=\frac{8}{15} E_{z i-1 / 2}^{n}+\frac{2}{3} e_{z i+1 / 4}^{n}-\frac{1}{5} e_{z i+3 / 4}^{n}, \\
& e_{z i-1 / 4}^{n}=-\frac{2}{63} E_{z i-3 / 2}^{n}+\frac{2}{3} E_{z i-1 / 2}^{n}+\frac{10}{21} e_{z i+1 / 4}^{n}-\frac{1}{9} e_{z i+3 / 4}^{n} .
\end{aligned}
$$

An alternative linear interpolation often used in AMR algorithms [2, 29], denoted here as Lin1,

$$
e_{z i-1 / 4}^{n}=E_{z i-1 / 2}^{n}+\frac{1}{8}\left[\frac{1}{2}\left(e_{z i+1 / 4}^{n}+e_{z i+3 / 4}^{n}\right)-E_{z i-3 / 2}^{n}\right] .
$$

We also examined two additional quadratic interpolants, denoted as Qu1 and Qu2 respectively,

$$
\begin{gathered}
e_{z i-1 / 4}^{n}=-\frac{1}{14} E_{z i-3 / 2}^{n}+\frac{5}{6} E_{z i-1 / 2}^{n}+\frac{5}{21} e_{z i+1 / 4}^{n}, \\
e_{z i-1 / 4}^{n}=-\frac{3}{32} E_{z i-3 / 2}^{n}+\frac{15}{16} E_{z i-1 / 2}^{n}+\frac{5}{32}\left[\left(e_{z i+1 / 4}^{n}+e_{z i+3 / 4}^{n}\right) / 2\right] .
\end{gathered}
$$

For the second sub-cycling step, ghost values have to be provided at time level $n+1 / 2$. For this task we have used the following four approaches for time interpolation.

The first approach, called here Lift, is based on Taylor expansion in time at the nearest coarse grid value $E_{z i-1 / 2}^{n}[2]$,

$$
e_{z i-1 / 2}^{n+1 / 2}=e_{z i-1 / 2}^{n}+\frac{1}{2}\left(\tilde{E}_{z i-1 / 2}^{n+1}-E_{z i-1 / 2}^{n}\right)
$$

where the $\tilde{E}_{z i-1 / 2}^{n+1}$ was obtained during the step (1) of the AMR algorithm. This coarse value is corrected during the last step, using the Yee scheme, when the updated fine interface values become available [29].

The second approach, called here Hav, is based on applying the standard Yee scheme to update the ghost value from time $n$ to time $n+1 / 2$,

$$
e_{z i-1 / 4}^{n+1 / 2}=e_{z i-1 / 4}^{n}+\nu_{x}\left(H_{y i}^{n+1 / 4}-H_{y i-1 / 2}^{n+1 / 4}\right)
$$


where the necessary magnetic field values are determined by using the following spacetime interpolations. Quadratic interpolation along the $y$-axis is used to determine $H_{y i-1}^{n \pm 1 / 2}$ and $H_{y i}^{n \pm 1 / 2}$. Then linear interpolation in time with weights $3 / 4$ and $1 / 4$ gives values at time level $n+1 / 4$. Finally, $H_{y i-1 / 2}^{n+1 / 4}$ is defined as the arithmetic average between $H_{y i-1}^{n+1 / 4}$ and $H_{y i}^{n+1 / 4}$.

The third approach applies the same spatial interpolation algorithms during each sub-cycling time step with the coarse values needed at time level $n+1 / 2$ computed as the arithmetic average between the coarse values at time levels $n$ and $n+1$.

The fourth approach we have examined is the previously published Collino algorithm [6]. It defines a global invariant $\mathcal{E}_{2}(n)$ over the whole domain including the grid interface. This single identity involves three unknown values $h_{y i}^{n+1 / 2}, h_{y i}^{n+1}$ and $H_{y i}^{n+1}$, without enforcing that the space-time collocated values $h_{y}^{n+1}$ and $H_{y i}^{n+1}$ are identical. These three unknown variables are denoted for short as $h_{1}, h_{2}$ and $H_{1}$. The global invariant is as follows

$$
0.5 *\left(h_{1}+h_{y}^{n} i\right) * e q 1+0.5 *\left(h_{2}+h_{1}\right) * e q 2-\left(H_{1}+H_{y i}^{n}\right) * e q 3=0,
$$

where

$$
\begin{aligned}
& e q 0=H_{1}+H_{y i}^{n}-0.5\left(h_{y i}^{n}+2 h_{1}+h_{2}\right), \\
& e q 1=e_{z i+1 / 4}^{n+1 / 4}+0.5 \nu\left(h_{1}-h_{y i}^{n}\right), \\
& e q 2=e_{z i+1 / 4}^{n+1 / 4}+\nu\left(h_{y i+1 / 2}^{n+1 / 2}-h_{1}\right)+0.5 \nu\left(h_{2}-h_{1}\right), \\
& e q 3=E_{z i-1 / 2}^{n+1 / 2}-0.5 \nu\left(H_{1}-H_{y i}^{n}\right) .
\end{aligned}
$$

To determine the three unknowns the global invariant is arbitrarily split into a system of three linear equations $e q 0=0, e q 1=e q 2=e q 3$. Many other choices for splitting are possible. For example, a "natural" choice would be to enforce the consistency of magnetic fields by setting $h_{2}=H_{1}$ and by damping the high numerical wavenumbers. We do not investigate these methods in this paper. In addition we investigate an algorithm, 1DWeil, reduced from the original 3D algorithm due to Weiland [22].

\begin{tabular}{|c|c|c|}
\hline Algorithm & Normal & Update to time $n+1 / 2$ \\
\hline \hline 1DLinLift & linear & Lift \\
\hline 1DLin1Lift & linear 1 & Lift \\
\hline 1DQuLift & quadratic & Lift \\
\hline 1DQu1Lift & quadratic 1 & Lift \\
\hline 1DQu2Lift & quadratic 2 & Lift \\
\hline 1DCuLift & cubic & Lift \\
\hline 1DQuHav & quadratic & Hav \\
\hline 1DLinLin & linear & linear \\
\hline 1DQuQu & quadratic & quadratic \\
\hline 1DQu1Qu1 & quadratic 1 & quadratic 1 \\
\hline 1DQu2Qu2 & quadratic 2 & quadratic 2 \\
\hline 1DCuCu & cubic & cubic \\
\hline
\end{tabular}

TABLE 3.1. Labeling of the one dimensional algorithms. 
3.1.1. 1D Algorithm labeling. The labels for the $1 \mathrm{D}$ algorithms, Table 3.1, are identified by prefix 1D followed by two parts: the spatial interpolation used in the direction normal to the interface during the first sub-cycling step and the type of space-time interpolation used during the second sub-cycling step. Some of the one dimensional algorithms used Lift or Hav during the second sub-cycling step, while others apply the same spatial interpolation algorithms during each sub-cycling time step with the coarse values needed at time level $n+1 / 2$ computed as the arithmetic average between the coarse values at time levels $n$ and $n+1$. For example, 1DQuQu uses quadratic spatial interpolation during both subcycling steps.
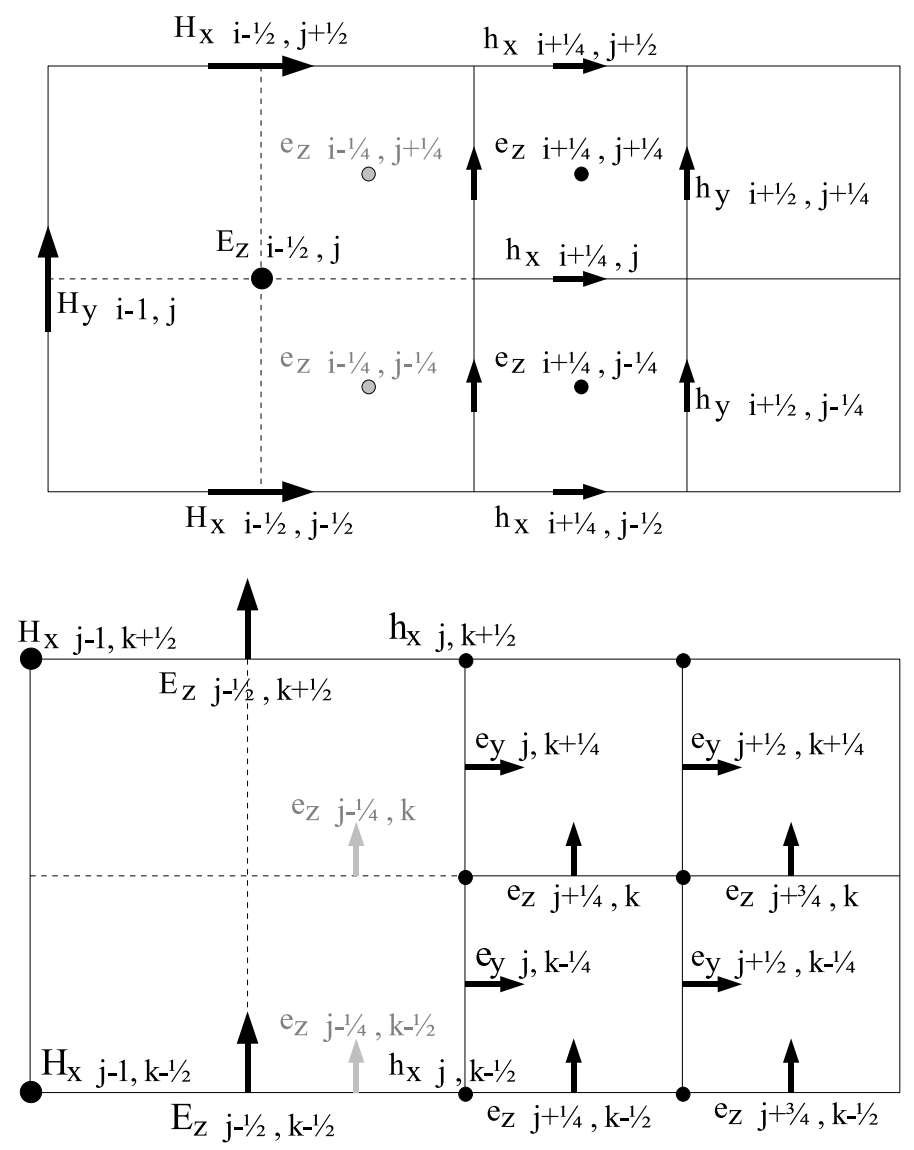

FIG. 3.1. TM coarse-fine grid interface (top) and TE coarse-fine grid interface (bottom) showing the location of the space-time interpolated ghost boundary values (greyscale).

3.2. Two dimensional interpolation algorithms. The two dimensional (TM) and (TE) cases are obtained from the full 3D staggered grids by projecting the $3 \mathrm{D}$ computational cell along the $z$ and $x$ directions respectively $[20,22,30]$. Two spatial interpolations are needed to determine the ghost boundary values, greyscale values in Fig. 3.1, before the refined grid may be advanced. One interpolation, tangential to the grid interface, involving only coarse grid values (upper case) and the other normal to the grid interface involving fine grid values (lower case). We 
study the stability of various combinations of interpolation routines when incorporated into to the 2D AMR algorithm presented in [29]. In addition we have investigated three previously published algorithms, $[22,6]$, denoted here as as TMWeil, TEWeil and Collino. The original Weil algorithm is a fully $3 \mathrm{D}$ algorithm that we reduced to its (TM) and (TE) projections, while the Collino algorithm is applicable only to one dimensional problems.

3.2.1. (TM) interpolation algorithms. The coarse-fine grid interface for the (TM) case, obtained by projecting the $3 \mathrm{D}$ grid onto an $\mathrm{x}-\mathrm{y}$ plane, is shown in Fig. 3.1 (left). For the TMWeil algorithm the coarse electric field values are defined at time levels $n-1 / 2$ and $n+1 / 2$, while the fine electric field is defined at time levels $n-1 / 4$, $n+1 / 4$ and $n+3 / 4$, and a linear interpolation is used in both directions,

$$
\begin{aligned}
E_{z i-1 / 2, j \pm 1 / 4}^{n+1 / 2} & =\frac{3}{4} E_{z i-1 / 2, j}^{n+1 / 2}+\frac{1}{4} E_{z i-1 / 2, j \pm 1}^{n+1 / 2}, \\
e_{z i-1 / 4, j \pm 1 / 4}^{n+1 / 4} & =\frac{2}{3} E_{z i-1 / 2, j \pm 1 / 4}^{n+1 / 2}+\frac{1}{3} e_{z i+1 / 4, j \pm 1 / 4}^{n+1 / 4} \\
e_{z i-1 / 4, j \pm 1 / 4}^{n+3 / 4} & =\frac{2}{3} E_{z i-1 / 2, j \pm 1 / 4}^{n+1 / 2}+\frac{1}{3} e_{z i+1 / 4, j \pm 1 / 4}^{n+3 / 4}
\end{aligned}
$$

This interpolation is only first order accurate in time. In our construction the electric field values are collocated in time differently from the TMWeil algorithm, the coarse electric field values are defined at time levels $n$ and $n+1$, while the fine electric field is defined at time levels $n, n+1 / 2$ and $n+1$. In the tangential direction both linear and quadratic interpolations, abbreviated as Lin and Qu respectively, were used:

$$
\begin{aligned}
E_{z i-1 / 2, j \pm 1 / 4}^{n} & =\frac{3}{4} E_{z i-1 / 2, j}^{n}+\frac{1}{4} E_{z i-1 / 2, j \pm 1}^{n}, \\
E_{z i-1 / 2, j \pm 1 / 4}^{n}=E_{z i-1 / 2, j}^{n} & +\frac{1}{8}\left(E_{z_{i-1 / 2, j+1}^{n}}^{n}-E_{z i-1 / 2, j-1}^{n}\right) \\
+ & \frac{1}{32}\left(E_{z_{i-1 / 2, j+1}^{n}}^{n}-2 E_{z i-1 / 2, j}^{n}+E_{z i-1 / 2, j-1}^{n}\right) .
\end{aligned}
$$

In the direction normal to the interface we applied linear, quadratic or cubic interpolations, denoted as $\mathrm{Lin}, \mathrm{Qu}$ and $\mathrm{Cu}$ respectively,

$$
\begin{aligned}
& e_{z i-1 / 4, j \pm 1 / 4}^{n}=\frac{2}{3} E_{z i-1 / 2, j \pm 1 / 4}^{n}+\frac{1}{3} e_{z i+1 / 4, j \pm 1 / 4}^{n}, \\
& e_{z i-1 / 4, j \pm 1 / 4}^{n}=\frac{8}{15} E_{z i-1 / 2, j \pm 1 / 4}^{n}+\frac{2}{3} e_{z i+1 / 4, j \pm 1 / 4}^{n}-\frac{1}{5} e_{z i+3 / 4, j \pm 1 / 4}^{n}, \\
& e_{z i-1 / 4, j \pm 1 / 4}^{n}=-\frac{2}{63} E_{z i-3 / 2, j \pm 1 / 4}^{n}+\frac{2}{3} E_{z i-1 / 2, j \pm 1 / 4}^{n}+\frac{10}{21} e_{z i+1 / 4, j \pm 1 / 4}^{n}-\frac{1}{9} e_{z i+3 / 4, j \pm 1 / 4}^{n},
\end{aligned}
$$

and two alternative quadratic interpolants, Qu1 and Qu2,

$$
\begin{aligned}
e_{z i-1 / 4, j \pm 1 / 4}^{n}= & -\frac{1}{14} E_{z i-3 / 2, j \pm 1 / 4}^{n}+\frac{5}{6} E_{z i-1 / 2, j \pm 1 / 4}^{n}+\frac{5}{21} e_{z i+1 / 4, j \pm 1 / 4}^{n}, \\
e_{z i-1 / 4, j \pm 1 / 4}^{n}=-\frac{3}{32} E_{z i-3 / 2, j \pm 1 / 4}^{n} & +\frac{15}{16} E_{z i-1 / 2, j \pm 1 / 4}^{n} \\
& +\frac{5}{32}\left[\left(e_{z i+1 / 4, j \pm 1 / 4}^{n}+e_{z i+3 / 4, j \pm 1 / 4}^{n}\right) / 2\right] .
\end{aligned}
$$


For the first sub-cycling step the fine field values are collocated in time with the coarse values, however, for the second sub-cycling step ghost values have to be provided at time level $n+1 / 2$. For this task we have used the following two approaches for time interpolation.

In the first approach we again use Lift, based on Taylor expansion in time at the nearest coarse grid value $E_{z i-1 / 2, j}^{n}[2]$,

$$
e_{z i-1 / 2, j \pm 1 / 4}^{n+1 / 2}=e_{z i-1 / 2, j \pm 1 / 4}^{n}+\frac{1}{2}\left(\tilde{E}_{z i-1 / 2, j}^{n+1}-E_{z i-1 / 2, j}^{n}\right),
$$

where the $\tilde{E}_{z i-1 / 2, j}^{n+1}$ was obtained during the step (1) of the AMR algorithm. This coarse value is corrected during the last step, using the Yee scheme, when the updated fine interface values become available [29].

The second approach, Hav, is based on applying the standard Yee scheme to update the ghost value from time $n$ to time $n+1 / 2$,

$$
\begin{aligned}
& e_{z i-1 / 4, j+1 / 4}^{n+1 / 2}=e_{z i-1 / 4, j+1 / 4}^{n}+\nu_{x}\left(H_{y i, j+1 / 4}^{n+1 / 4}-H_{y i-1 / 2, j+1 / 4}^{n+1 / 4}\right) \\
& -\nu_{y}\left(H_{x i-1 / 4, j+1 / 2}^{n+1 / 4}-H_{x i-1 / 4, j}^{n+1 / 4}\right) \text {, }
\end{aligned}
$$

where the necessary magnetic field values are determined by using the following space-time interpolations. Quadratic interpolation along $y$-axis is used to determine $H_{y i-1, j \pm 1 / 4}^{n \pm 1 / 2}$ and $H_{y i, j \pm 1 / 4}^{n \pm 1 / 2}$. Then linear interpolation in time with weights $3 / 4$ and $1 / 4$ gives values at time level $n+1 / 4$. Finally, $H_{y i-1 / 2, j \pm 1 / 4}^{n+1 / 4}$ is defined as the arithmetic average between $H_{y i-1, j \pm 1 / 4}^{n+1 / 4}$ and $H_{y i, j \pm 1 / 4}^{n+1 / 4}$. The $x$-components of the coarse magnetic field at levels $j \pm 1 / 2$ are computed using quadratic interpolation normal to the grid interface,

$$
h_{x i-1 / 4, j \pm 1 / 2}^{n+1 / 4}=\frac{8}{15} H_{x i-1 / 2, j \pm 1 / 2}^{n+1 / 4}+\frac{2}{3} h_{x i+1 / 4, j \pm 1 / 2}^{n+1 / 4}-\frac{1}{5} h_{x i+3 / 4, j \pm 1 / 2}^{n+1 / 4} .
$$

The required values at time level $n+1 / 4$ are determined by linear time interpolation from known values at time levels $n \pm 1 / 2$. Finally $H_{x i-1 / 4, j}^{n+1 / 4}$ is defined as

$$
H_{x i-1 / 4, j}^{n+1 / 4}=\frac{1}{2}\left(\begin{array}{c}
n+1 / 4 \\
x i-1 / 4, j+1 / 2
\end{array}+H_{x i-1 / 4, j-1 / 2}^{n+1 / 4}\right) .
$$

3.2.2. (TE) interpolation algorithms. The coarse-fine grid interface for the (TE) case, obtained by projecting the 3D grid onto a $\mathrm{y}-\mathrm{z}$ plane, is shown in Fig. 3.1 (right). In this case the middle value $E_{z i-1 / 2, j}^{n}$ is determined by an arithmetic average,

$$
E_{z j-1 / 2, k}^{n}=\frac{1}{2}\left(E_{z j-1 / 2, k-1 / 2}^{n}+E_{z j-1 / 2, k+1 / 2}^{n}\right) .
$$

In the direction normal to the interface we investigated the same interpolation schemes as in the (TM) case. For the Lift algorithm the time gradient needed at point $(j-1 / 2, k)$ is computed as an arithmetic average of the time gradients at points $(j-1 / 2, k \pm 1 / 2)$. The Hav approach is slightly simpler for the (TE) case as the values $H_{x j-1, k \pm 1 / 2}^{n+1 / 4}$ and $H_{x j, k \pm 1 / 2}^{n+1 / 4}$ require time interpolation only from known values $H_{x j-1, k \pm 1 / 2}^{n \pm 1 / 2}$ and $H_{x j, k \pm 1 / 2}^{n \pm 1 / 2}$. The middle value $H_{x j-1 / 2, k}^{n+1 / 4}$ is computed as an arithmetic average of the four corner values $H_{x j-1, k \pm 1 / 2}^{n+1 / 4}$ and $H_{x j, k \pm 1 / 2}^{n+1 / 4}$. 
3.2.3. 2D algorithm labeling. The labels for the (TM) algorithms, Table 3.2 , consist of three parts: the spatial interpolations used in the directions tangential and normal to the interface during the first sub-cycling step and the type of space-time interpolation used during the second sub-cycling step. For example, the algorithm labeled as TMLinQuLift corresponds to the (TM) algorithm with linear (tangential) and quadratic (normal) interpolation during the first sub-cycling step and the Lift algorithm for second sub-cycling step. For the (TE) case, Table 3.3, only normal interpolation is needed during the first sub-cycling step and consequently the notation shortens, with the middle value obtained from equation 3.1. For example the algorithm labeled as TEQuHav denotes an algorithm employing quadratic interpolation in the normal direction and using Hav for the second sub-cycling step.

\begin{tabular}{|c|c|c|c|}
\hline Algorithm & Tangential & Normal & Update to time $n+1 / 2$ \\
\hline \hline TMLinLinLift & linear & linear & Lift \\
\hline TMLinQuLift & linear & quadratic & Lift \\
\hline TMLinQu1Lift & linear & quadratic 1 & Lift \\
\hline TMLinCuLift & linear & cubic & Lift \\
\hline TMQuLinLift & quadratic & linear & Lift \\
\hline TMQuQuLift & quadratic & quadratic & Lift \\
\hline TMQuQu1Lift & quadratic & quadratic 1 & Lift \\
\hline TMQuCuLift & quadratic & cubic & Lift \\
\hline TMQuQuHav & quadratic & quadratic & Hav \\
\hline
\end{tabular}

TABLE 3.2. Labeling of the two dimensional (TM) algorithms.

\begin{tabular}{|c|c|c|}
\hline Algorithm & Normal & Update to time $n+1 / 2$ \\
\hline \hline TELinLift & linear & Lift \\
\hline TEQuLift & quadratic & Lift \\
\hline TEQu1Lift & quadratic 1 & Lift \\
\hline TECuLift & cubic & Lift \\
\hline TEQuHav & quadratic & Hav \\
\hline
\end{tabular}

TABLE 3.3. Labeling of the two dimensional (TE) algorithms.

\section{Stability tests for AMR FDTD algorithms}

Two tests based on the GKS-Trefethen theory $[9,8,23,24,25]$ were performed. These tests are necessary conditions for stability, however, passing these two tests is not sufficient to assert the stability of the algorithm. The first test calculates reflection/transmission coefficients for the plane wave incident on the grid interface from either the fine or the coarse grid side. A reflection coefficient larger than unity will manifest itself in a trapping instability as the respective wave packet amplitudes will increase on each reflection. Similar instability will occur as the wave travels across a periodic layered domain if the product between transmission coefficients of different layers is larger than unity. An "infinite" reflection coefficient corresponds to Kreiss modes that represent mode-boundary resonance. When the value of the reflection coefficient is equal to unity, further tests for resonant behavior are needed as the possibility of mode-mode resonance still remains. The second stability test 
checks for growing modes (eigensolutions) on an infinite domain with a single grid interface allowing for both propagating and evanescent modes. This test will detect the exponentially growing modes with $\operatorname{Im}(\omega)<0$ or Kreiss modes, that are limiting cases of such modes, where $\operatorname{Im}(\omega)=0,[8]$. Mode-mode resonance and growth may occur when frequencies or wavenumbers of two modes coincide. A simple illustration of this behavior is given by a leap-frog scheme that has an evolution matrix of the Jordan block form, $A=\left(\begin{array}{ll}1 & 1 \\ 0 & 1\end{array}\right)$ and $A^{n}=\left(\begin{array}{ll}1 & n \\ 0 & 1\end{array}\right)$. The spatial mode-mode resonance, with coinciding wavenumber, leads to growth in space and possibly in time, on a finite domain with periodic or perfectly reflecting boundaries. Both of these cases require further investigation.

4.1. Reflection and transmission through a single interface in one dimension. Reflection and transmission coefficients presented in this section were computed in two ways, analytically using Mathematica symbolic manipulations and numerically using a $\mathrm{C}$ code implementations of the AMR algorithms. The analytical solutions were obtained by substituting a plane wave ansatz into the interface boundary conditions and solving the resulting linear system. The plane wave ansatz takes into account that a single coarse grid frequency $\omega \Delta t$ generates two fine grid plane waves with fine grid wavenumbers corresponding to the primary frequency $\omega \Delta t$ and secondary aliased frequency $\tilde{\omega} \Delta t=\omega \Delta t+2 \pi$. The respective wavenumbers are denoted as $k(\omega \Delta t)$ and $\tilde{k}(\tilde{\omega} \Delta t)$. The primary plane wave is a propagating wave only for grid frequencies $\omega \Delta t \in\left[0,4 \sin ^{-1}(\nu)\right]$ while the secondary aliased wave is evanescent for all grid frequencies $\omega \Delta t \in[0, \pi]$. The exact solution is obtained using the following plane wave ansatz,

$$
\begin{aligned}
& \left(\begin{array}{l}
e_{z} \\
h_{y}
\end{array}\right)=\left(\begin{array}{l}
1 \\
1
\end{array}\right) e^{i(k x+\omega t)}+R\left(\begin{array}{r}
1 \\
-1
\end{array}\right) e^{i(-k x+\omega t)}+\tilde{R}\left(\begin{array}{r}
1 \\
-1
\end{array}\right) e^{i(-\tilde{k} x+\tilde{\omega} t)}, \\
& \left(\begin{array}{l}
E_{z} \\
H_{y}
\end{array}\right)=T\left(\begin{array}{l}
1 \\
1
\end{array}\right) e^{i(k x+\omega t)}
\end{aligned}
$$

for an incident wave impinging from the fine grid side $x>0$, and

$$
\begin{aligned}
& \left(\begin{array}{l}
e_{z} \\
h_{y}
\end{array}\right)=T\left(\begin{array}{r}
1 \\
-1
\end{array}\right) e^{i(-k x+\omega t)}+\tilde{T}\left(\begin{array}{r}
1 \\
-1
\end{array}\right) e^{i(-\tilde{k} x+\tilde{\omega} t)}, \\
& \left(\begin{array}{c}
E_{z} \\
H_{y}
\end{array}\right)=\left(\begin{array}{r}
1 \\
-1
\end{array}\right) e^{i(-k x+\omega t)}+R\left(\begin{array}{l}
1 \\
1
\end{array}\right) e^{i(k x+\omega t)}
\end{aligned}
$$

for an incident wave impinging from the coarse grid side $x<0$.

Analytically, the reflection and transmission coefficients are solutions of a linear system of equations obtained by substitution of the solution ansatz into the interface boundary conditions. For example, for the 1DWeil algorithm the resulting interface equations are,

$$
\begin{aligned}
& H_{y i}^{n}=h_{y i}^{n}, \\
& e_{z i-1 / 4}^{n+1 / 4}=\frac{2}{3} E_{z i-1 / 2}^{n+1 / 2}+\frac{1}{3} e_{z i+1 / 4}^{n+1 / 4}, \\
& e_{z i-1 / 4}^{n+3 / 4}=\frac{2}{3} E_{z i-1 / 2}^{n+1 / 2}+\frac{1}{3} e_{z i+1 / 4}^{n+3 / 4} .
\end{aligned}
$$

The first equation defines the coarse interface magnetic field in terms of the collocated fine magnetic field, while last two equations provide the boundary values of the electric field for the refined region. 
Numerically measured reflection and transmission coefficients were computed using an incident Gaussian pulse [29]. The reflection and transmission coefficients computed analytically (continuous line) and numerically (dashed patches) are in excellent agreement as illustrated in the Fig. 4.1. The reflection and transmission coefficients for fine-to-coarse propagation for three algorithms with $R \leq 1$, 1DQuLift, 1DQuHav and 1DWeil, are shown in Fig. 4.1. At low frequencies the algorithms recover second order accuracy with the reflection coefficient $R \sim O\left(\Delta t^{2}\right)$, while Collino (not shown) and 1DWeil algorithms have $R \sim O(\Delta t)$ near zero frequency. Note that even though the transmission coefficient at the cutoff frequency is larger than unity, the product of $\left|T_{f c}\right|\left|T_{c f}\right|$ is less than unity, and there is no build up of the energy in the high frequency modes.
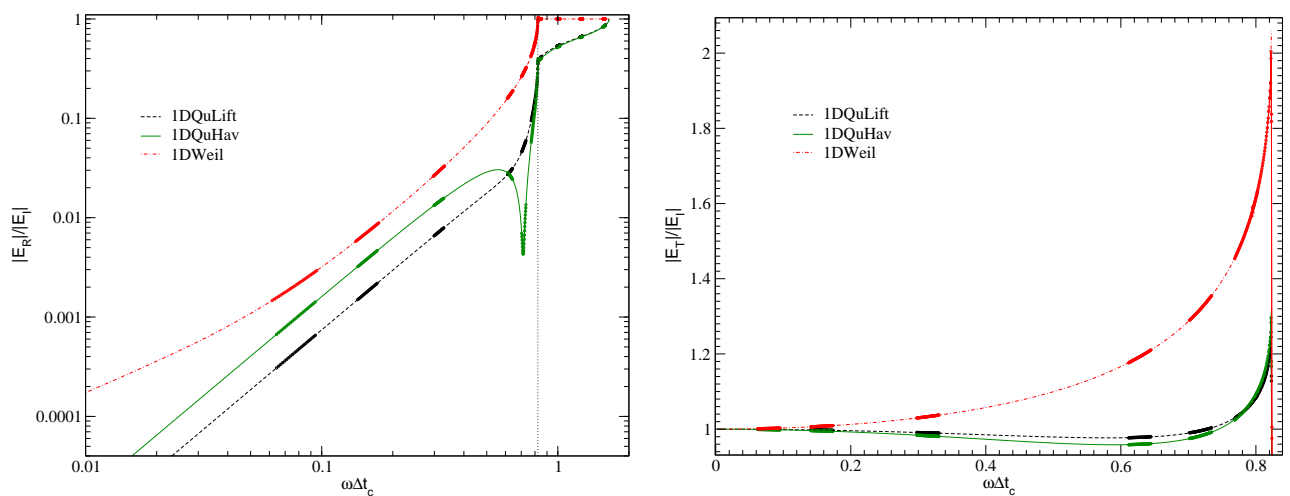

FIG. 4.1. Fine-to-coarse reflection (left) and transmission (right) coefficients for the algorithms 1DQuLift, 1DQuHav and 1DWeil. Analytical (continuous lines) and numerical (dashed patches) solutions are over plotted.

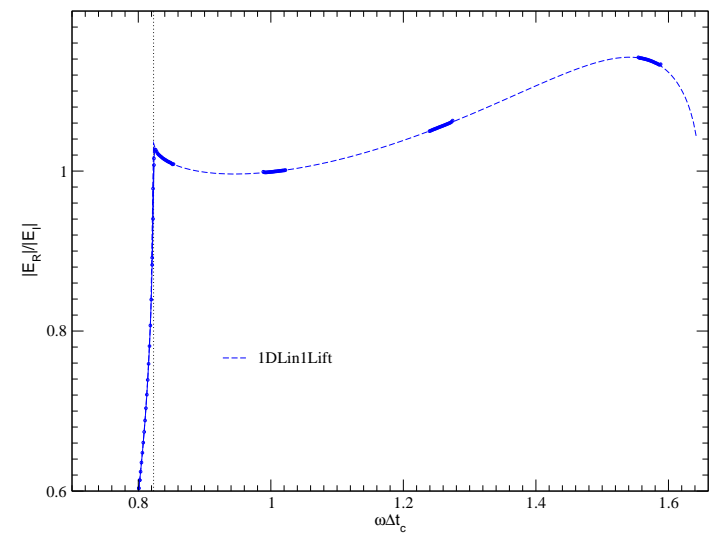

FIG. 4.2. Fine-to-coarse reflection coefficient for the unstable, $R>1$, 1DLin1Lift algorithm, for frequencies between fine and coarse grid cutoff. Analytical (continuous lines) and numerical (dashed patches) solutions are over plotted.

The reflection coefficient for the Collino algorithm has the same behavior as the 1DWeil algorithm, where the reflection coefficient is unity for the frequencies between 
the coarse and fine cutoff, $\left[2 \sin ^{-1}(\nu), 4 \sin ^{-1}(\nu)\right]$, with a possibility of resonance of high frequency numerical modes. In the Fig. 4.2 we plot the absolute value of the reflection coefficient for an unstable algorithm 1DLin1Lift [2]. Note that this algorithm is stable in the context of AMR algorithms for the Euler equations of fluid dynamics due to the dissipative nature of the numerical schemes employed.
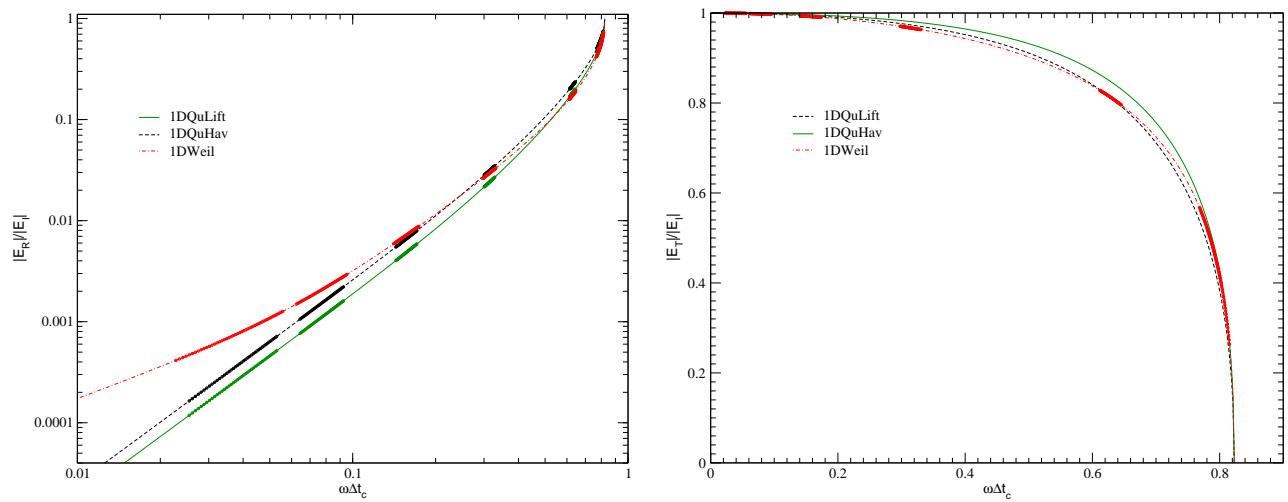

FIG. 4.3. Coarse-to-fine reflection (left) and transmission (right) coefficients for the algorithms 1DQuLift, 1DQuHav and 1DWeil. Analytical (continuous lines) and numerical (dashed patches) solutions are over plotted.

For completeness we also show, in Fig. 4.3, the reflection and transmission coefficients for coarse-to-fine incidence for the algorithms, 1DQuLift, 1DQuHav and 1DWeil. Note again that the slopes near zero frequency indicate second order accuracy for the 1DQuLift and 1DQuHav algorithms and first order for the 1DWeil algorithm. Table 4.1 summarizes the reflection coefficients for fine-to-coarse incidence for the rest of the one dimensional algorithms discussed in the previous section.

\begin{tabular}{|l|lll|}
\hline$R>1$ & 1DLin1Lift & 1DLinLin & 1DQuQu \\
& 1DCuCu & 1DQu2Lift & 1DQu2Qu2 \\
\hline \multirow{3}{*}{$R \leq 1$} & 1DLinLift & 1DQuLift & 1DQu1Lift \\
& 1DCuLift & 1DQu1Qu1 & 1DQuHav \\
& 1DWeil & Collino & \\
\hline
\end{tabular}

TABLE 4.1. Reflection coefficients for fine-to-coarse incidence.

4.2. Modes of the infinite domain with a single interface in one dimension. Modes of the infinite domain with a single grid interface can be computed using a modification of the solution ansatz used in the previous section. This ansatz consists of outgoing waves only and includes both propagating and evanescent waves.

The solution ansatz is as follows,

$$
\begin{aligned}
& \left(\begin{array}{l}
e_{z} \\
h_{y}
\end{array}\right)=C\left(\begin{array}{r}
1 \\
-1
\end{array}\right) e^{i(-k x+\omega t)}+\tilde{C}\left(\begin{array}{r}
1 \\
-1
\end{array}\right) e^{i(-\tilde{k} x+\tilde{\omega} t)}, \\
& \left(\begin{array}{l}
E_{z} \\
H_{y}
\end{array}\right)=D\left(\begin{array}{l}
1 \\
1
\end{array}\right) e^{i(k x+\omega t)}
\end{aligned}
$$


Substituting it into the three interface boundary conditions gives a homogeneous linear system for three unknown wave amplitudes $C, \tilde{C}$ and $D$. For a nonzero solution to exist the determinant of the system must be zero. This is the same as the determinant condition of GKS stability theory $[9,8]$. The determinant is a nonlinear function of the complex variable $\omega \Delta t$. Its zeroes are investigated graphically by plotting a surface of the absolute value of the determinant. The graphical analysis was supplemented by local minimization and secant method root finding in cases where a zero occurs on the imaginary axis $\operatorname{Im}(\omega \Delta t)=0$. The surface plots for all the cases considered indicate that the absolute value of the determinant rapidly grows away from the imaginary axis in the half plane $\operatorname{Im}(\omega \Delta t)<0$, thus eliminating the exponentially growing modes. The numerical frequencies considered are in the range $[0,2 \pi]$, with $2 \pi$ being the Nyquist frequency on the fine grid since normalization is done using coarse grid spacing. In Fig. 4.4 we show the surface plot and $\operatorname{Im}(\omega \Delta t)=0$ cut of the $|\operatorname{Det}(\omega \Delta t)|$ for the Collino algorithm. Note, that the surface is a discontinuous function near $\operatorname{Im}(\omega \Delta t=0)$, but the mode for $\omega \Delta t=\pi$ is present for all three limiting cases, $\operatorname{Im}(\omega \Delta t)<0, \operatorname{Im}(\omega \Delta t)=0$ (shown) and $\operatorname{Im}(\omega \Delta t)>0$.
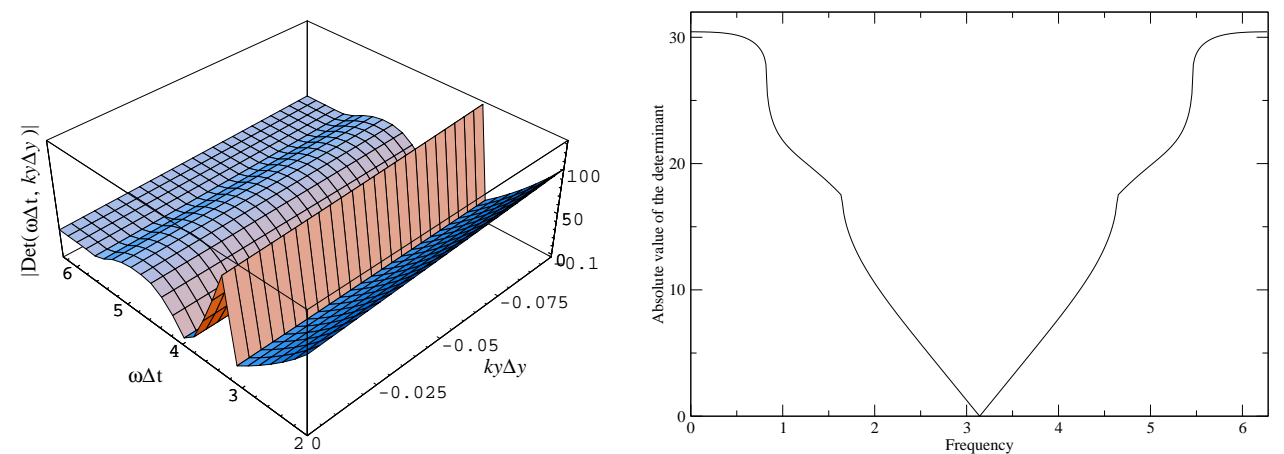

FIG. 4.4. Surface (left) and $\operatorname{Im}(\omega \Delta t)=0$ cut (right) of absolute value of the determinant for the Collino algorithm.

The Collino algorithm has a mode at $\omega \Delta t=\pi$. In this case both $k_{x} \Delta x$ and the aliased wavenumber $\tilde{k}_{x} \Delta x$ coincide. Therefore, the general solution of the finite difference equation has the form $e^{i k \Delta x j}, j e^{i k \Delta x j}$, where $j$ is the spatial index. On an infinite domain with zero boundary conditions such modes decay in space. On the other hand, if such modes persist on a finite domain with zero boundary conditions, solutions growing linearly in space, and thus in time, may also be invoked. Whether such a scenario of mode-mode resonance on the finite domain will be realized has to be investigated further. None of the other algorithms showed the existence of modes for $\operatorname{Im}(\omega \Delta t) \leq 0$.

4.3. Reflection and transmission through a single interface in two dimensions. In two dimensions a single coarse frequency, $\omega \Delta t$, and a single transverse wavenumber, $k_{y} \Delta y$, correspond to two fine grid frequencies $\omega \Delta t$ and $\omega \Delta t+2 \pi$, and two transverse wavenumbers $k_{y} \Delta y$ and $k_{y} \Delta y+2 \pi$. The coarse and fine dispersion relations then determine the wavenumbers normal to the interface, $k_{x} \Delta x$ and $k_{x}^{p, q}, p, q=0,1$, where the signs are chosen according to the sign selection rule dis- 
cussed in section 2 ,

$$
\begin{gathered}
\sin ^{2}\left(\frac{\omega \Delta t}{2}\right)=\nu^{2}\left[\sin ^{2}\left(\frac{k_{x} \Delta x}{2}\right)+\sin ^{2}\left(\frac{k_{y} \Delta y}{2}\right)\right] \\
\sin ^{2}\left(\frac{\omega \Delta t+2 \pi p}{4}\right)=\nu^{2}\left[\sin ^{2}\left(\frac{k_{x}^{p, q} \Delta x}{4}\right)+\sin ^{2}\left(\frac{k_{y} \Delta y+2 \pi q}{4}\right)\right] p, q=0,1 .
\end{gathered}
$$

The five unknown coefficients in the solution ansatz for the reflection/transmission problem exactly correspond to the number of interface boundary conditions. For example, a coarse interface magnetic field defines a single reflected/transmitted wave on the coarse grid, while two fine ghost values at each sub-cycling time correspond to four reflected or transmitted waves on the fine grid. To introduce the solution ansatz we first define the amplitude normalization in terms of the following variables,

$$
\begin{aligned}
& A=\nu \frac{\sin \left(k_{x} \Delta x / 2\right)}{\sin ((\omega \Delta t) / 2)}, \quad B=-\nu \frac{\sin \left(\left(k_{y} \Delta y\right) / 4\right)}{\sin ((\omega \Delta t) / 4)}, \\
& a_{p, q}=\nu \frac{\sin \left(k_{x}^{p, q} \Delta x / 4\right)}{\sin ((\omega \Delta t+2 \pi p) / 4)}, \quad b_{p, q}=\nu \frac{\sin \left(\left(k_{y} \Delta y+2 \pi q\right) / 4\right)}{\sin ((\omega \Delta t+2 \pi p) / 4)} .
\end{aligned}
$$

Then, the plane wave solution ansatz is

$$
\left(\begin{array}{c}
e_{z} \\
h_{y} \\
h_{x}
\end{array}\right)=\left(\begin{array}{c}
1 \\
a_{0,0} \\
-b_{0,0}
\end{array}\right) e^{i\left(k_{x}^{0,0} x+k_{y} y+\omega t\right)}+\sum_{p, q} R_{p, q}\left(\begin{array}{c}
1 \\
-a_{p, q} \\
-b_{p, q}
\end{array}\right) e^{i\left(-k_{x}^{p, q} x+k_{y} y+\omega t\right)},
$$

with $p=0,1$ and $q=0,1$. The transmitted plane wave on the coarse side has the form

$$
\left(\begin{array}{l}
E_{z} \\
H_{y} \\
H_{x}
\end{array}\right)=T e^{i\left(k_{x} x+k_{y} y+\omega t\right)}\left(\begin{array}{r}
1 \\
A \\
-B
\end{array}\right)
$$

Similarly, when the wave impinges from the coarse side, $x<0$, there are four possible transmitted waves. The incident and reflected plane wave solution ansatz on the coarse side is,

$$
\left(\begin{array}{c}
E_{z} \\
H_{y} \\
H_{x}
\end{array}\right)=\left(\begin{array}{r}
1 \\
-A \\
-B
\end{array}\right) e^{i\left(-k_{x} x+k_{y} y+\omega t\right)}+R\left(\begin{array}{r}
1 \\
A \\
-B
\end{array}\right) e^{i\left(k_{x} x+k_{y} y+\omega t\right)} .
$$

On the fine side the transmitted wave ansatz is as follows,

$$
\left(\begin{array}{c}
e_{z} \\
h_{y} \\
h_{x}
\end{array}\right)=\sum_{p, q} T_{p, q} e^{i\left(-k_{x}^{p, q} x+k_{y} y+\omega t\right)}\left(\begin{array}{c}
1 \\
-a_{p, q} \\
-b_{p, q}
\end{array}\right) e^{i\left(-k_{x}^{p, q} x+k_{y} y+\omega t\right)},
$$

where as before each of the variables $p$ and $q$ are equal to 0 or 1 . 

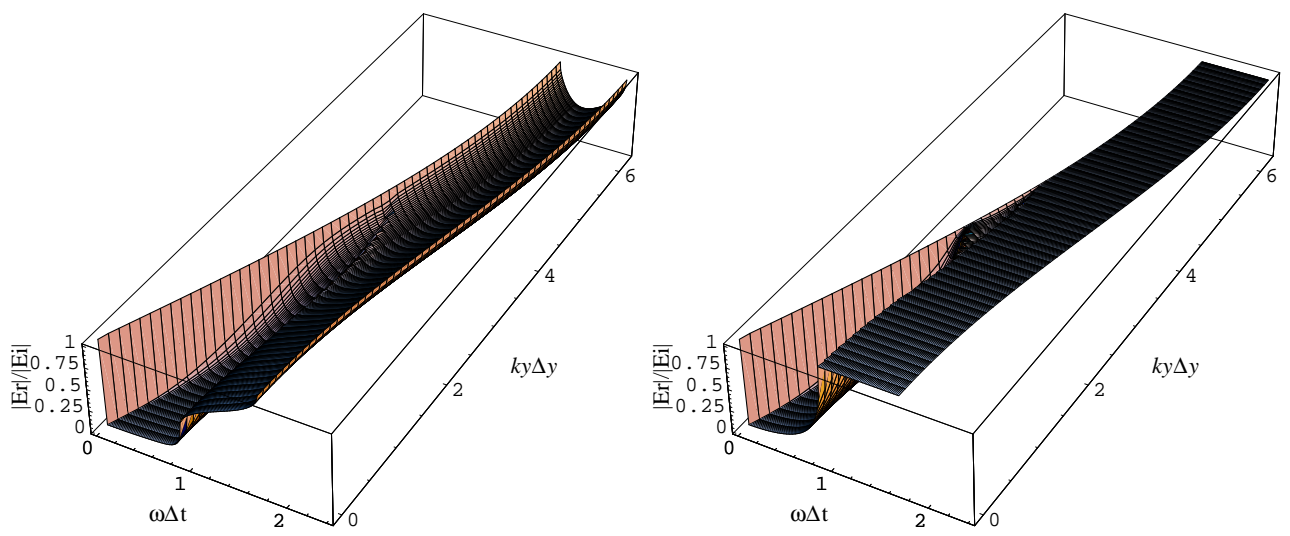

FIG. 4.5. The (TM) reflection coefficient for fine-to-coarse incidence for the TMQuQuLift (left), and TMWeil (right) algorithms.

4.3.1. (TM) algorithms. For the (TM) case all the algorithms that had $R \leq 1$ in the one dimensional case also preserved, $R \leq 1$, for both fine-to-coarse and coarse-to-fine incidence. A surface plot of the absolute value of the reflection coefficients as function of frequency and transverse wavenumber, $\left|R\left(\omega \Delta t, k_{y} \Delta y\right)\right|$ for two representative cases, the TMQuQuLift and TMWeil algorithms, are shown in Fig. 4.5.

The transverse wavenumber $k_{y} \Delta y$ runs through interval $[0,2 \pi]$ since both fine and coarse wavenumbers were normalized by the coarse grid size $\Delta y$. The frequency interval is determined for each transverse wavenumber by the condition that, for the incident wave, $k_{x}^{0,0}$ must be real, i.e. $0 \leq \sin \left(k_{x}^{0,0} \Delta x\right) \leq 1$.

In Fig. 4.6 the surface plots of the absolute value of the reflection coefficient for coarse-to-fine incidence for TMQuQuLift and TMWeil algorithms are shown.
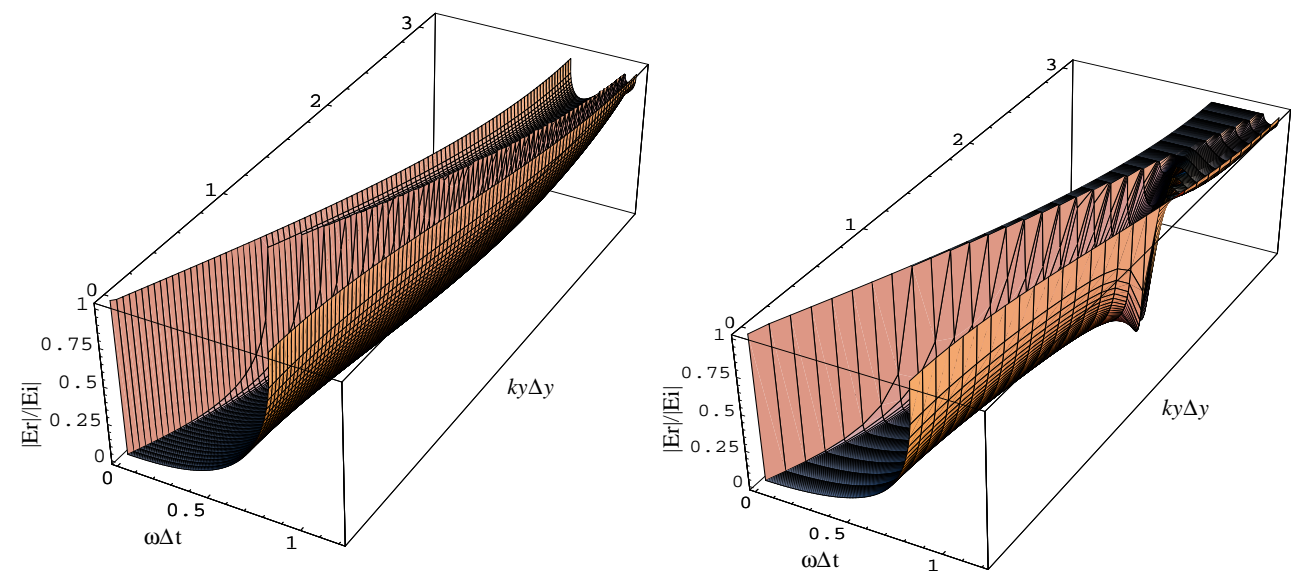

FIG. 4.6. The (TM) reflection coefficient for coarse-to-fine incidence for the TMQuQuLift (left), and TMWeil (right) algorithms.

Note, in our previous publication [29] the sign of the imaginary part of the 
wavenumber $k_{x}$ was chosen incorrectly, consequently the reflection coefficient as plotted in Fig. 2.6 (left) of [29] incorrectly shows $R>1$. The correct reflection coefficient is shown in Fig. 4.6 (left), and has $R<1$ over all $\left(\omega \Delta t, k_{y} \Delta y\right)$ space. We compared the absolute values of several numerically computed reflection coefficients with analytical solutions for coarse-to-fine incidence. The numerical reflection coefficients were computed using an incident plane wave on a domain with periodic boundary conditions in the direction along the interface, and PML boundary conditions in the direction normal to the interface. Modulation of the numerically generated plane wave was less than one percent due to initial turn-on of the source. The resulting numerical and analytical reflection coefficients are shown in the Table 4.2 .

\begin{tabular}{|c|c|c|c|}
\hline$k_{y} \Delta$ & $\omega \Delta t$ & Numerical & Analytical \\
\hline \hline$\pi$ & 0.85 & 0.785 & 0.789 \\
\hline$\pi$ & 0.83 & 0.880 & 0.874 \\
\hline$\pi / 2$ & 0.6 & 0.857 & 0.865 \\
\hline$\pi / 2$ & 0.85 & 0.306 & 0.308 \\
\hline$\pi / 2.5$ & 0.49 & 0.890 & 0.892 \\
\hline$\pi / 2.5$ & 0.6 & 0.130 & 0.130 \\
\hline
\end{tabular}

TABLE 4.2. Comparison of the numerically and analytically computed absolute values of the reflection coefficients for coarse-to-fine incidence for the TMQuQuLift algorithm.

In Fig. 4.7 we present one dimensional cuts for the TMQuQuLift algorithm as a function of $\omega \Delta t$ along the three planes, $k_{y} \Delta y=\pi, \pi / 2, \pi / 2.5$, for coarse-to-fine incidence together with several reflection coefficients obtained numerically.

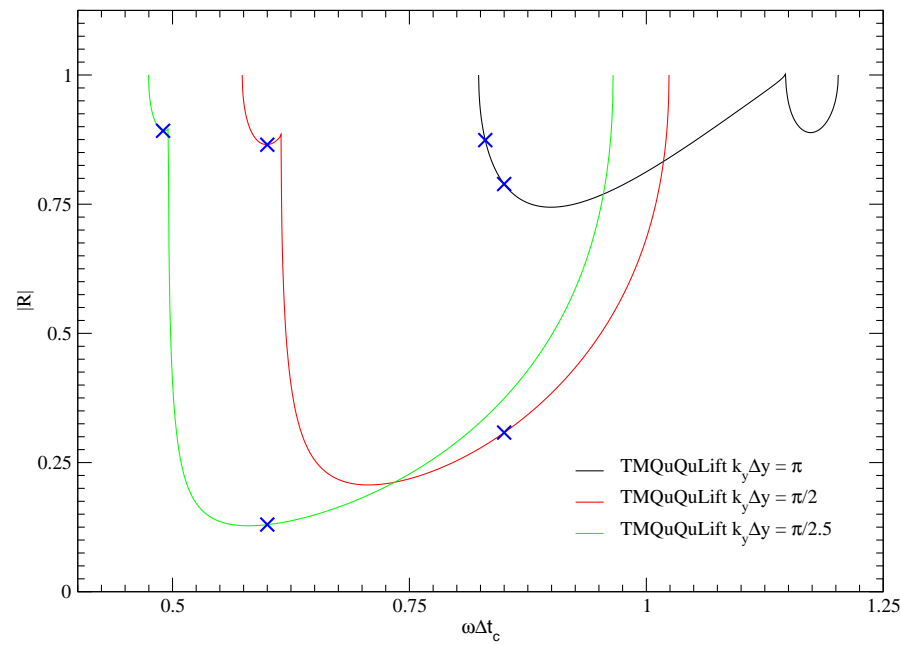

FIG. 4.7. One dimensional cuts of analytically computed reflection coefficients for the TMQuQuLift algorithm for coarse-to-fine incidence along the three planes $k_{y} \Delta y=\pi, \pi / 2, \pi / 2.5$ (continuous line) overploted with the numerically computed reflection coefficients ( $X$ symbols).

\subsection{2. (TE) algorithms.}

For coarse-to-fine incidence in the (TE) case, all algorithms were unstable, $R>1$, except TEQuHav and TEWeil which had $R \leq 1$. Surface plots of the absolute value 

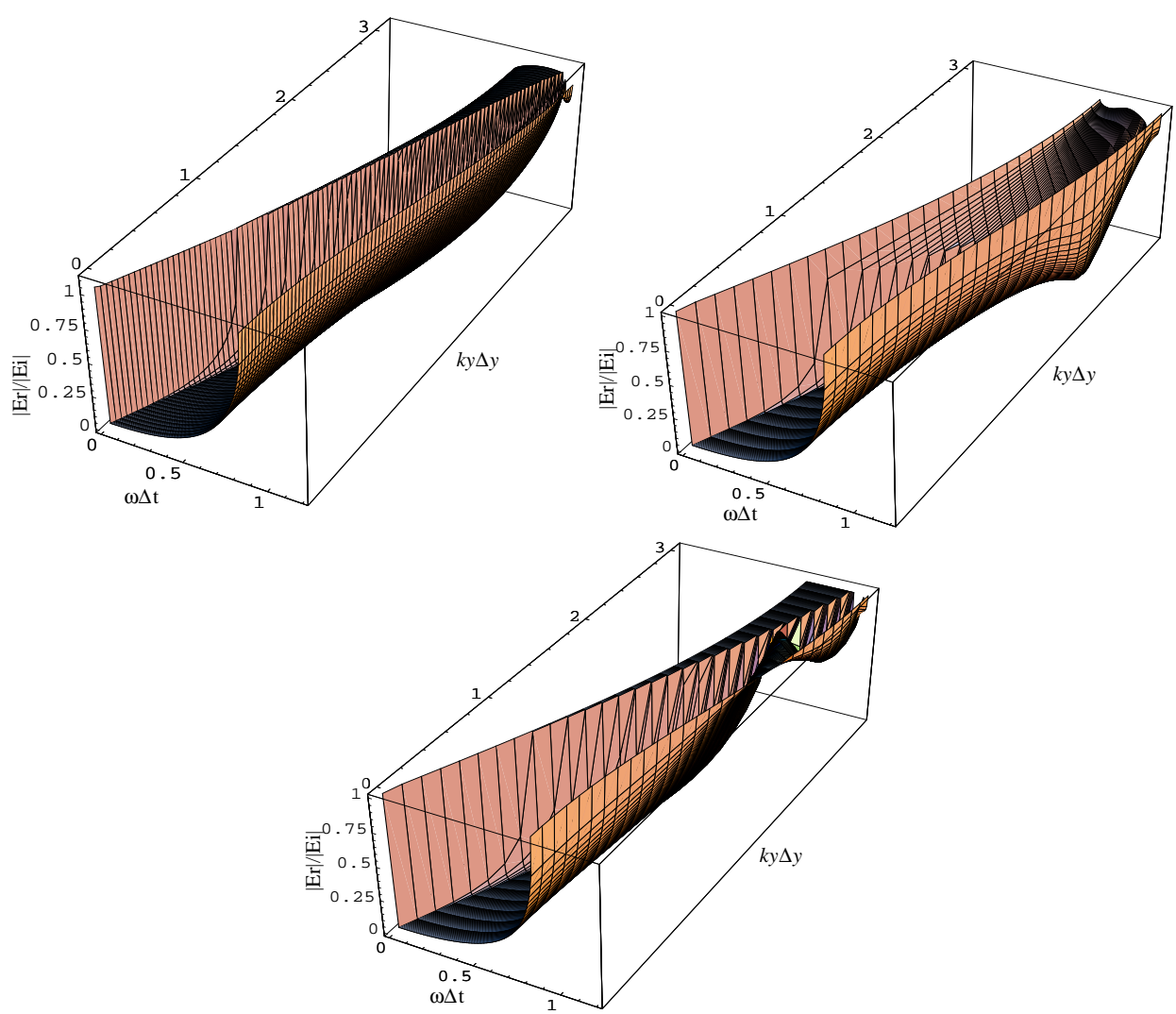

FIG. 4.8. The (TE) reflection coefficient for coarse-to-fine incidence TEQuLift (top left), TEQuHav (top right) and TEWeil (bottom) algorithms.

of the reflection coefficients for the TEQuLift, TEQuHav and TEWeil algorithms are shown in Fig. 4.8.

In the (TE) case when the incident wave approaches from the fine side all the algorithms, except TEWeil and TEQuHav have some region with $R>1$ in $\left(\omega \Delta t, k_{y} \Delta y\right)$ space. Surface plots of the absolute value of the reflection coefficients for TEQuLift, TEQuHav and TEWeil algorithms are shown in Fig. 4.9.

Similar to the (TM) case we compared the absolute values of several numerically computed reflection coefficients with analytical solutions for coarse-to-fine incidence for both TEQuHav and TEQuLift algorithms. The resulting numerical and analytical reflection coefficients are shown in the Tables 4.3 and 4.4. In Fig. 4.10 we present one dimensional cuts for TEQuHav and TEQuLift algorithms as a function of $\omega \Delta t$ along the planes, $k_{y} \Delta y=0, \pi / 6, \pi / 2$ and $k_{y} \Delta y=\pi / 6, \pi / 2$, respectively, for coarse-tofine incidence together with several reflection coefficients obtained numerically.

\subsection{Stability of modes on the infinite domain with a single interface.}

In this section we examine the existence of modes for six algorithms TMQuLinLift, TMQuQu1Lift, TMQuQuHav, TMWeil, TEQuHav, TEWeil that passed the $R<1$ stability test, and two algorithms TELinLift, TEQu1Lift that failed the $R<1$ stability test.

Modes of the infinite domain with a single grid interface can be computed using a 

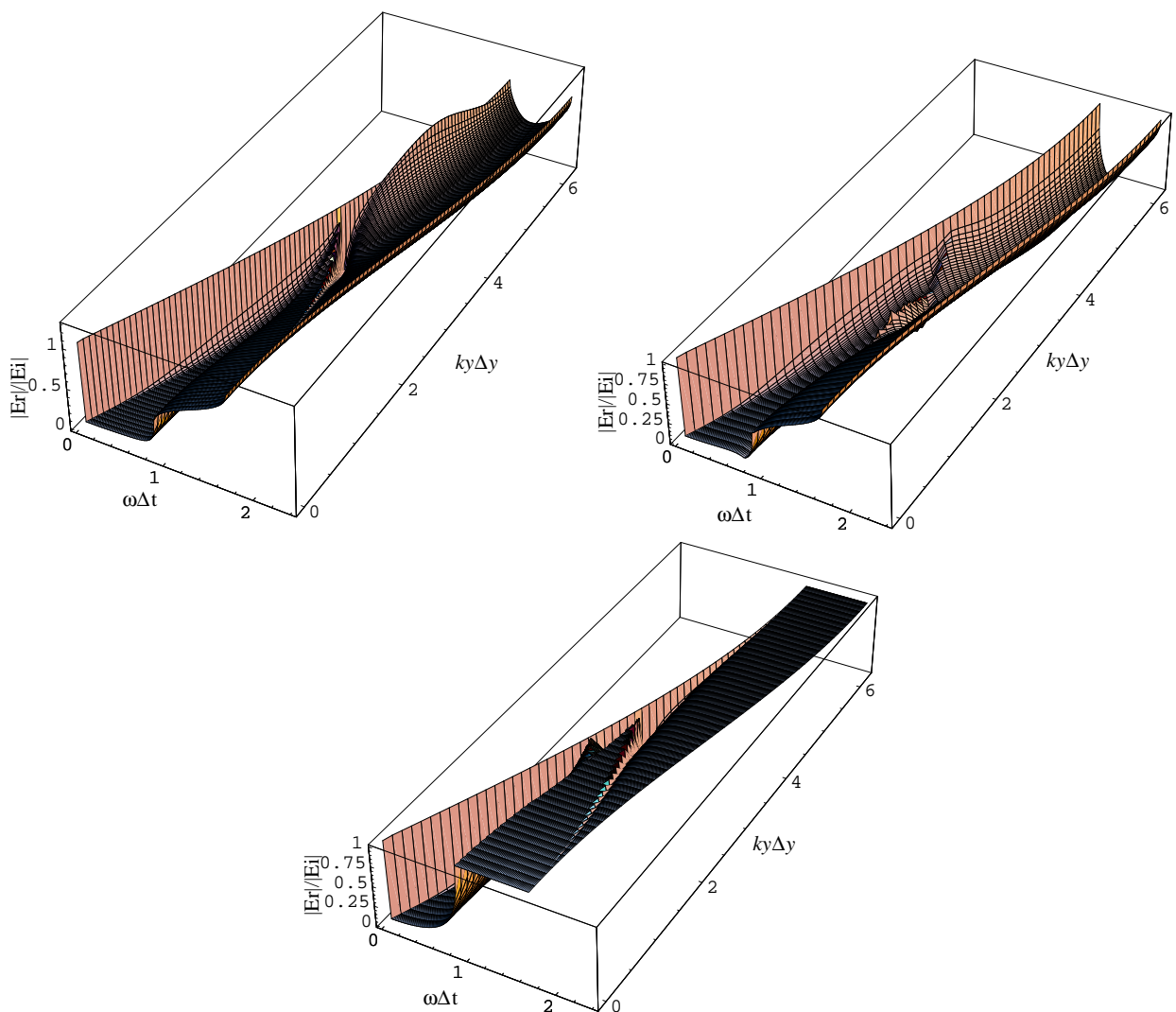

FIG. 4.9. The (TE) reflection coefficient for fine-to-coarse incidence TEQuLift (top left), TEQuHav (top right) and TEWeil (bottom) algorithms.

\begin{tabular}{|c|c|c|c|}
\hline$k_{y} \Delta$ & $\omega \Delta t$ & Numerical & Analytical \\
\hline \hline 0 & 0.6 & 0.155 & 0.156 \\
\hline 0 & 0.3 & 0.0214 & 0.0215 \\
\hline 0 & 0.8 & 0.606 & 0.605 \\
\hline$\pi / 6$ & 0.3 & 0.0247 & 0.0251 \\
\hline$\pi / 6$ & 0.6 & 0.143 & 0.144 \\
\hline$\pi / 2$ & 0.6 & 0.835 & 0.833 \\
\hline$\pi / 2$ & 0.8 & 0.237 & 0.236 \\
\hline
\end{tabular}

TABLE 4.3. Comparison of the numerically and analytically computed absolute values of the reflection coefficients for coarse-to-fine incidence for TEQuHav algorithm.

modification of the solution ansatz used in the previous section. This ansatz consists of outgoing waves only and includes both propagating and evanescent waves. The solution ansatz is as follows,

$$
\left(\begin{array}{l}
E_{z} \\
H_{y} \\
H_{x}
\end{array}\right)=F\left(\begin{array}{r}
1 \\
A \\
-B
\end{array}\right) e^{i\left(k_{x} x+k_{y} y+\omega t\right)},
$$




$$
\left(\begin{array}{l}
e_{z} \\
h_{y} \\
h_{x}
\end{array}\right)=\sum_{p, q} G_{p, q}\left(\begin{array}{c}
1 \\
-a_{p, q} \\
-b_{p, q}
\end{array}\right) e^{i\left(-k_{x}^{p, q} x+k_{y} y+\omega t\right)}
$$

with the mode amplitude normalization defined as before by equations 4.1 and 4.2.

The domain is periodic in the transverse direction, $y$, and is infinite in the normal direction, $x$. Both evanescent and propagating waves are considered by allowing for complex frequency $\omega \Delta t$ and complex normal wavenumbers $k_{x}$ and $k_{x}^{p, q}$, while the transverse wavenumber $k_{y} \Delta y$ is assumed to be always real. Substituting this ansatz into the interface boundary relations gives a homogeneous system of five linear equations for five unknowns amplitudes. Setting the determinant of this linear system to zero gives a nonlinear equation that determines the frequencies of the mode. Zeroes of this equation are studied numerically by plotting the absolute values of the determinant, $\left|\operatorname{Det}\left(\omega \Delta t, k_{y} \Delta y\right)\right|$, for $\operatorname{Im}(\omega) \leq 0$. When the modes were detected they were further examined by cuts in the vicinity of the imaginary axis in the half-plane $\operatorname{Im}(\omega<0)$ in order to check for possible nearby exponentially growing modes. Since the graphs for all four algorithms showed rapid increase of the determinant away from the axis in the direction of exponentially growing modes, $\operatorname{Im}(\omega \Delta t)<0$, the search was limited to the real axis itself. Local minimization and secant root finding routines were used to double check the results of the graphical investigation. An example of a sur-

\begin{tabular}{|c|c|c|c|}
\hline$k_{y} \Delta$ & $\omega \Delta t$ & Numerical & Analytical \\
\hline \hline$\pi / 6$ & 0.3 & 0.0261 & 0.0266 \\
\hline$\pi / 6$ & 0.6 & 0.171 & 0.171 \\
\hline$\pi / 2$ & 0.6 & 1.0464 & 1.0465 \\
\hline$\pi / 2$ & 0.8 & 0.243 & 0.243 \\
\hline
\end{tabular}

TABLE 4.4. Comparison of the numerically and analytically computed absolute values of the reflection coefficients for coarse-to-fine incidence for TEQuLift algorithm.

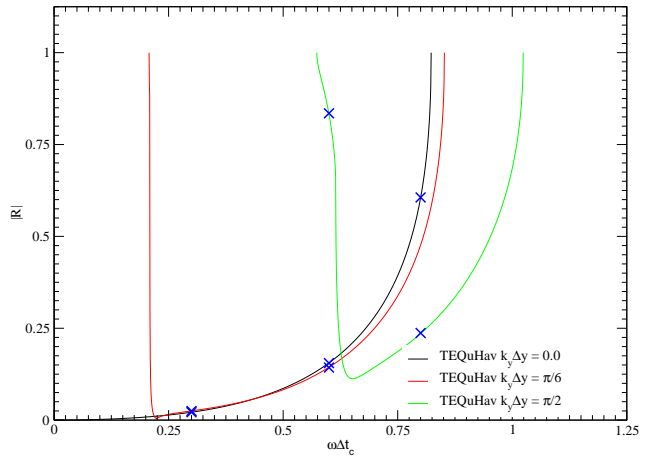

FIG. 4.10. One dimensional cuts of analytically computed reflection coefficients for the TEQuHav and TEQuLift algorithms for coarse-to-fine incidence along the three planes $k_{y} \Delta y=0, \pi / 6, \pi / 2$ and $k_{y} \Delta y=\pi / 6, \pi / 2$, respectively, (continuous line) overplotted with the numerically computed reflection coefficients (X symbols). 
face plot showing that there are no zeroes of the determinant for the TMQuQu1Lift algorithm for the (TM) mode for real $\omega$ is shown in Fig. 4.11.

Similar mode behavior for both (TM) and (TE) cases was shown by all algorithms, except the TMWeil and TEWeil. Both of these algorithms admitted modes on the imaginary axis, $\operatorname{Im}(\omega \Delta t=0)$, for $k_{y} \Delta y=\pi$ and $k_{y} \Delta y=2 \pi$, corresponding to the coarse and fine transverse Nyquist wavenumbers, respectively. These modes vanished under the perturbation away from the imaginary axis, therefore excluding the presence of the exponentially growing modes. Therefore, the only possibility left is the presence of mode-mode resonances. The mode-interface resonances were excluded by the absence of the infinite reflection coefficients for TMWeil and TEWeil algorithms as discussed in the previous section. In Fig. 4.12 we show a cut of the $\left|\operatorname{Det}\left(\omega \Delta t, k_{y} \Delta y\right)\right|$

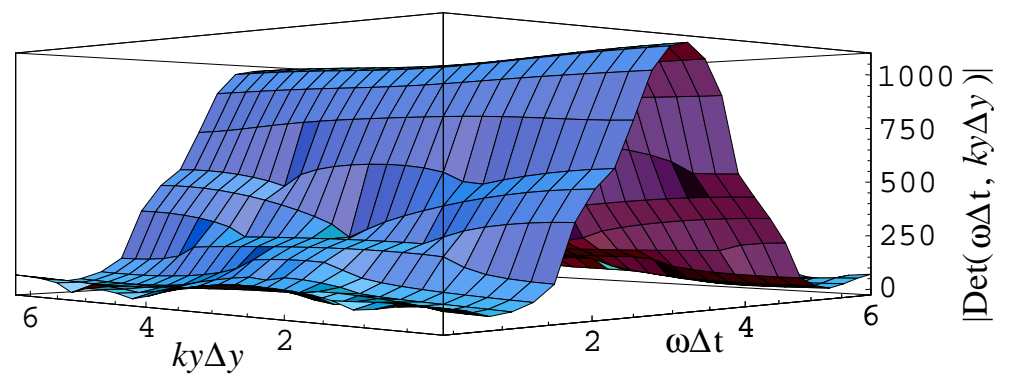

FIG. 4.11. Surface plot for the absolute value of the determinant for the TMQuQu1Lift algorithm.

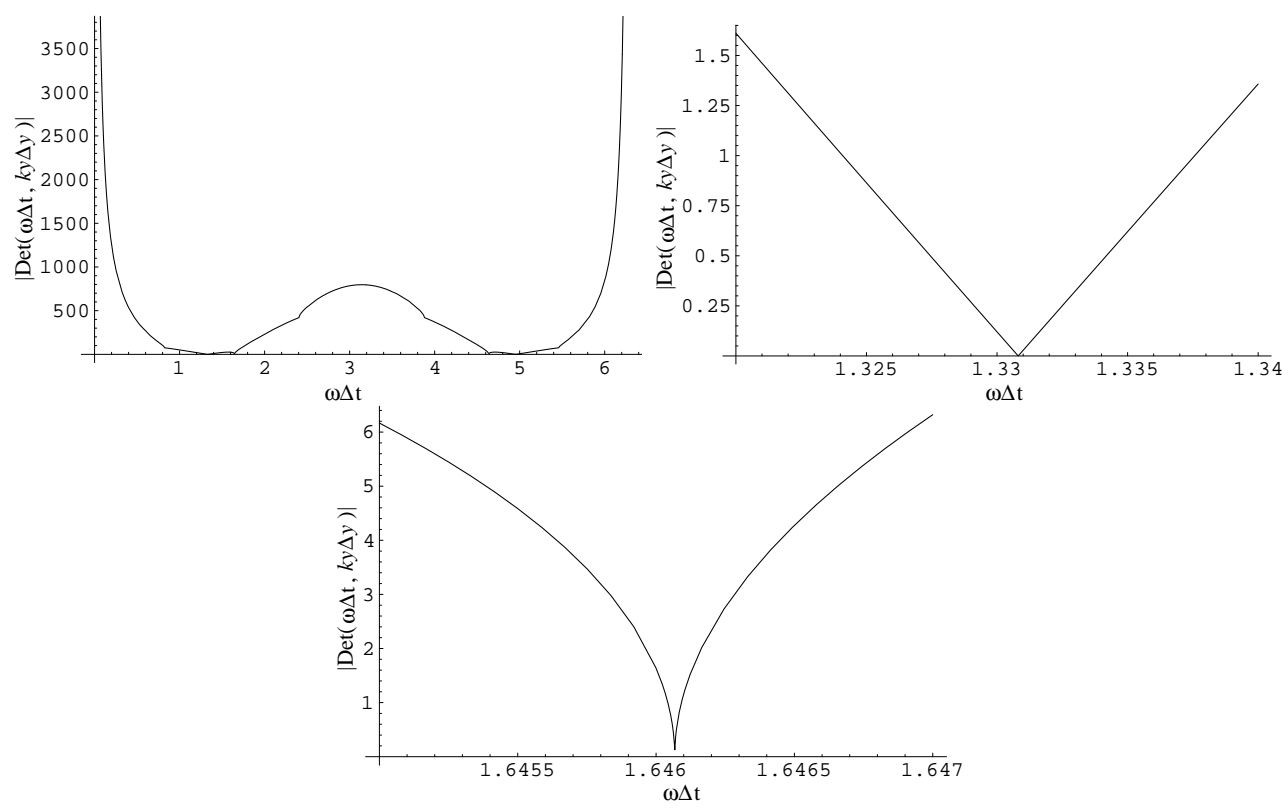

FIG. 4.12. Cut along the imaginary axis of the absolute value of the determinant for the TEWeil algorithm (left) together with the close-up of the graph near the two zeroes located in the interval [1, 2]. 
along the real axis of the frequency for the (TE) case when $k_{y} \Delta y=2 \pi$, as a function of the grid frequency $\omega \Delta t$ over an interval $[0,2 \pi]$.

Whether such borderline modes may lead to instability on a finite domain will be investigated numerically in the next section.
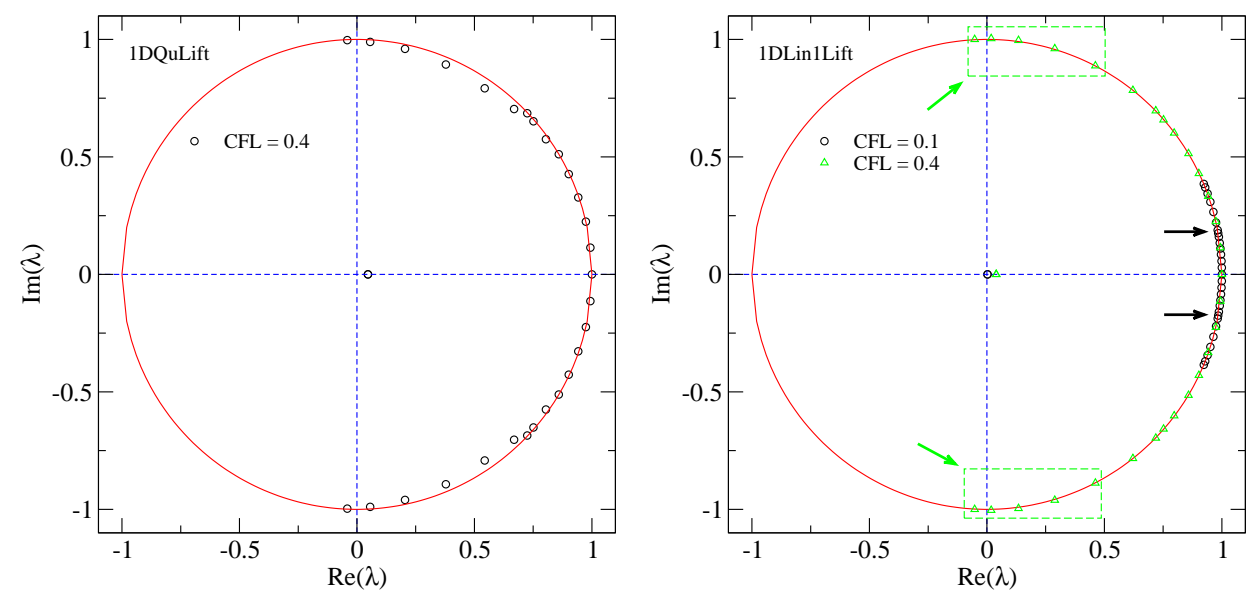

FIG. 4.13. Distribution of the eigenvalues on the complex plane for methods 1DQuLift and 1DLin1Lift. Dashed boxes and arrows indicate eigenvalues that fall outside of the unit circle, $|\lambda|>1$.
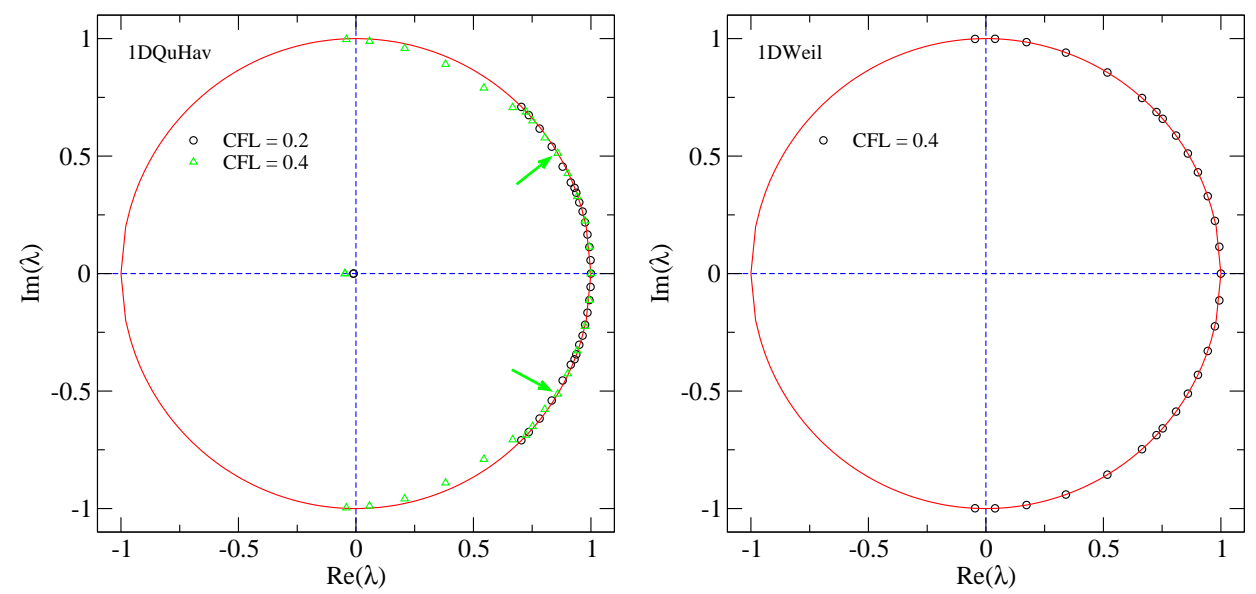

FIG. 4.14. Distribution of the eigenvalues on the complex plane for methods 1DQuHav and 1DWeil. Arrows indicate eigenvalues that fall outside of the unit circle, $|\lambda|>1$.

4.5. Matrix stability analysis of modes on the finite domain. In this section we apply a matrix stability analysis to a fully discrete problem on a finite domain with a single refinement patch. We present the results for a particular domain as a function of the CFL number. This analysis provides a necessary and sufficient condition for stability on a given grid. It states that the method is stable iff the update matrix has eigenvalues lying inside or on the unit circle. In addition, eigenvalues lying on the unit circle should be non-defective. Implemented numerically, this is 
equivalent to determining if an eigenvector subspace is smaller than the eigenvalue algebraic multiplicity (defective matrix), i.e. whether the canonical form of a matrix contains non-trivial Jordan blocks. This problem is ill-conditioned due to its sensitivity to perturbations and requires an extrapolation procedure from a series of high precision computations [12]. In the one-dimensional case considered below, however, this situation, arising from coinciding eigenvalues on the unit circle, was not encountered. Note, that this analysis is more general than the spectral radii analysis, as the latter is inconclusive about the coinciding eigenvalues on the unit circle, which require consideration of the dimension of the corresponding eigenspace to rule out the mode resonances. Eigenvalues of the update matrix were evaluated numerically, using the ARPACK iterative eigensolver [14].

4.5.1. One-dimensional matrix stability analysis. In this subsection, we consider the following one-dimensional algorithms 1DQuLift, 1DLin1Lift, 1DQuHav and 1DWeil as described in Section 4.1. The solution state vector is represented by the $\mathbf{E}_{f}, \mathbf{H}_{f}$ field values on the fine grid and the $\mathbf{E}_{c}, \mathbf{H}_{c}$ field values in the regions of the coarse grid that do not overlap with the fine grid. Algorithms 1DQuLift, 1DLin1Lift and 1DQuHav require magnetic field values for the update of the ghost cells, and therefore the coarse $\mathrm{H}$-field values collocated with the fine $\mathrm{H}$-fields at the grid interfaces are also included as part of the solution vector.

We consider solutions of an eigenvalue problem resulting from a discretization based on a single fine grid patch of $8 \Delta_{f}\left(=4 \Delta_{c}\right)$ cells centered on a $10 \Delta_{c}$ coarse grid. Reflecting boundary conditions $(\mathbf{E}=0)$ are applied on the coarse grid. The numerical tolerance for the error in the computed eigenvalues was set to $10^{-10}$.

Fig. 4.13 and Fig. 4.14 show the computed eigenvalue distributions in the complex plane. Algorithm 1DLin1Lift results in several eigenvalues (indicated in Fig. 4.13 by the arrows) that fall just outside of the unit circle. Fig. 4.15 shows that with a decreasing Courant number the maximum of the eigenvalue magnitude approaches unity from above, $\max |\lambda| \rightarrow 1^{+}$.

Algorithm 1DQuLift has $\max |\lambda| \leq 1$, with the simple eigenvalue $\lambda=(1,0)$ having the largest magnitude for any CFL values $\nu<1$. Similar behavior characterizes algorithm 1DQuHav which, however, requires a smaller CFL value to move all the eigenvalues inside of the unit circle.

Algorithm 1DWeil results in simple eigenvalues located on the unit circle, independent of the CFL number, similar to the FDTD scheme without the grid refinement.

The above results obtained from the matrix analysis are in agreement with the necessary conditions for stability from the reflection/transmission analysis for the one-dimensional methods, derived in section 4.1. In addition, the analysis indicates that there are no coinciding eigenvalues of unit magnitude, for which an eigenvector subspace is smaller than the eigenvalue algebraic multiplicity, which can lead to the existence of growing modes and instability. Numerical simulations performed with random noise initial data indicate damping, amplification or non-dissipative behavior for each of the algorithms, in accord with the corresponding eigenvalue distributions.

Stability analysis based on the computation of the spectral radius of a matrix, resulting from a semi-discrete [28] or a fully-discrete problem [31], allows evaluation of the stability condition structure and dependence on the mesh size. Figure 4.15(right) shows the dependence of the stabilty number, (an inverse of the Courant number $\left.=\Delta x / c_{\text {light }} \Delta t\right)$, on the grid step $\Delta x$. It characterizes the effects of both spatial and time discretizations and was computed numerically for a problem with one level of grid refinement and open domain boundaries. We considered the 1DQuLift and 
1DQuHav interpolation methods, both of which have second order accuracy in time and space. The stabilty number converges from below to a constant value of $1.41 \approx \sqrt{2}$ (corresponding to a CFL $c_{\text {light }} \Delta t / \Delta x=0.708$ ), when $\Delta x$ changes over approximately two orders of magnitude. For CFL numbers above the limiting value of 0.708 , we have found that the stability condition is not a monotone function and depends strongly on the time step.
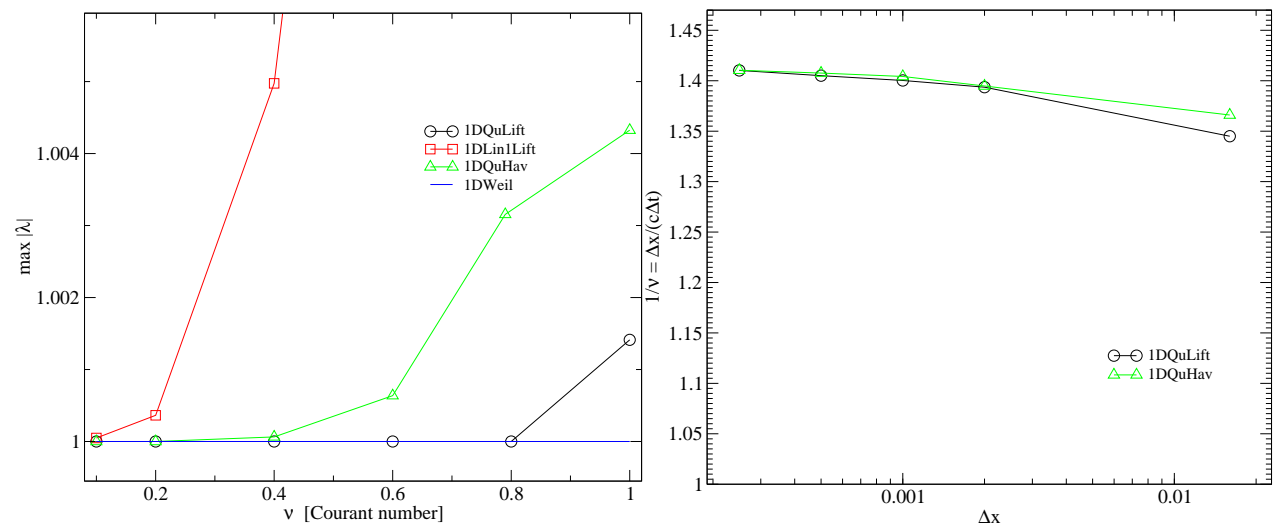

FIG. 4.15. Left: Dependence of the maximum of the eigenvalue magnitude on the Courant number for methods 1DQuLift, 1DLin1Lift, 1DQuHav and 1DWeil. Right: Stability condition as a function of mesh size for methods 1DQuLift and 1DQuHav.
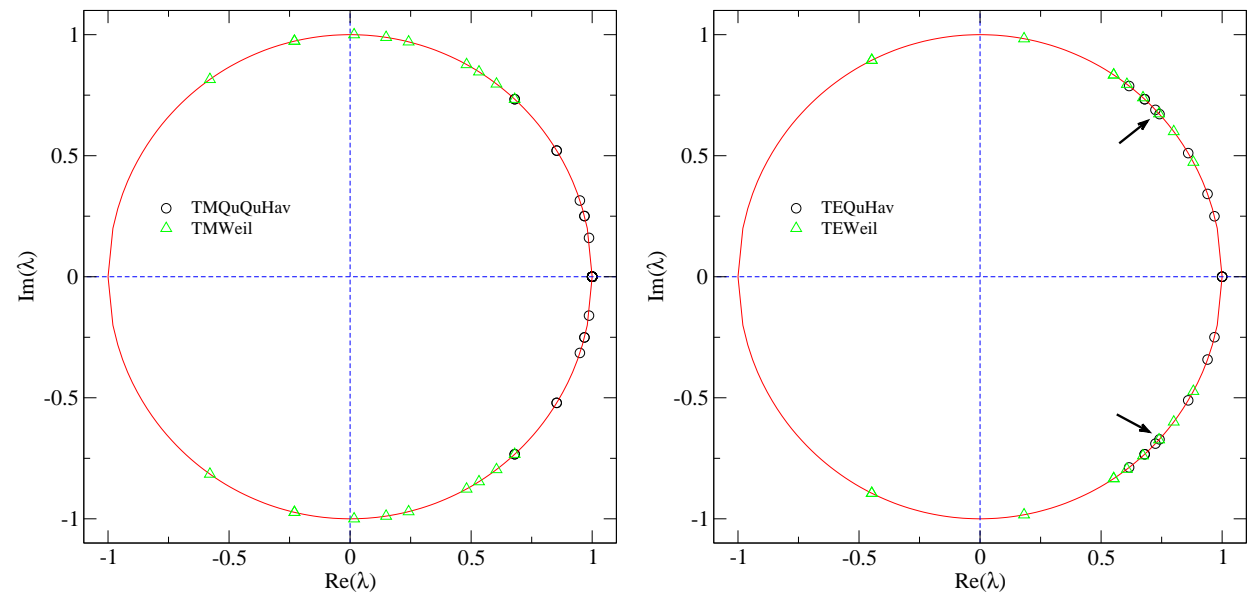

FIG. 4.16. Distribution of twenty largest (in magnitude) eigenvalues on the complex plane for TEQuHav, TMQuQuHav, TEWeil and TMWeil algorithms computed on a symmetric two-level grid with $n_{c}=10, n_{f}=8 C F L=0.4$. For the TEQuHav algorithm arrows indicate the complex-conjugate eigenvalue pair that fall outside of the unit circle, $|\lambda|>1$.

4.5.2. Two-dimensional matrix stability analysis. In this subsection the eigenvalues of the update matrix of the TEQuHav, TMQuQuHav, TEWeil and TMWeil algorithms are considered. The problem is discretized on an $n_{c} \times n_{c}$ coarse grid containing 
one $n_{f} \times n_{f}$ fine grid patch, with reflecting boundary conditions $(\mathbf{E}=0)$ applied on the coarse grid.

For $C F L=0.4$, the maximum eigenvalue magnitude computed for the TMQuQuHav algorithm is found to be within the unit circle for all values of $n_{c}$ and $n_{f}$ that were considered, except for the case of $n_{f}=4$ and $\bmod \left(n_{c}, 2\right)=0$, with symmetric placement of the fine grid with respect to the coarse grid center. This arrangement leads to one or more pairs of complex-conjugate eigenvalues with $|\lambda|>1$. On the same grid structure with smaller Courant numbers (e.g. $C F L=0.2$ ), these eigenvalues move inside of the unit circle, $|\lambda| \leq 1$.

For $C F L=0.4$ and the TEQuHav algorithm, eigenvalues with $|\lambda|>1$ exist for the case of $n_{f}=4$ and a placement of the fine grid within one grid of the coarse grid center. Additional modes with $|\lambda|>1$ exist in the case of $n_{f}>4$ and a symmetric arrangement of the fine grid with respect to the coarse grid center. For the case of the algorithms TEQuHav, TMQuQuHav, TEWeil and TMWeil algorithms Fig. 4.16 shows eigenvalue distributions in the complex-plane for the case of a refined patch, $n_{f}=8$, placed in the center of the coarse grid, $n_{c}=10$.

\section{Examples of Numerical Instabilities}

In this section we illustrate the stable/unstable behavior of various algorithms. Grid configurations are chosen to highlight trapping instabilities on both coarse and fine patches. A CFL number of 0.4 was used in all of our examples.

Example 1. (TM) Trapping instability on a single fine grid refinement patch.

In this example, we illustrate trapping instability for the (TM) case using the TMQuLin1Lift algorithm on a computational domain containing a single refinement patch. For comparison we illustrate stable behavior of the TMQuQuHav algorithm on the same computational domain. The coarse domain contains $40 \times 40$ uniformly spaced points with a grid resolution of $\Delta z=\Delta y=20 \mathrm{~nm}$. A single refined patch consisting of 30 fine cells in the z-direction is positioned in the center of the computational domain. The boundary conditions were periodic in the y-direction and PML boundary conditions were implemented in the z-direction. All fields were initialized to zero and a plane wave source was placed in the coarse grid at the top of the coarse domain. The $E_{x}$ field component is driven to produce a plane wave impinging onto the coarseto-fine interface at 30 degrees with an amplitude normalized to one at a wavelength $\lambda=400 \mathrm{~nm}$.

The onset of the trapping instability on the fine patch for the TMQuLin1Lift is shown in Fig. 5.1 (left) after $2 \times 10^{4}$ iterations with the final time being $520 \mathrm{fs}$. The performance of the TMQuQuHav algorithm did not show any instability after $2 \times 10^{4}$ iterations, as shown in Fig. 5.1 (right), in agreement with the theory.

Example 2. (TE) Trapping instability on the coarse patch placed between two fine refinement patches.

To show the agreement with the instability analysis of the previous section, in this example, we illustrate the trapping instability for the (TE) TEQuLift algorithm on a computational domain consisting of two refinement patches surrounding a coarse strip placed in the middle of the computational domain. The width of each fine patch and the middle coarse patch are 10 coarse (20 fine) grid points. The spatial resolution and boundary conditions are the same as in the previous example. The $H_{x}$ field was initialized in the middle of the computational domain, $z=0$ to produce a plane wave impinging onto the coarse-to-fine interface from inside the coarse patch at an angle 

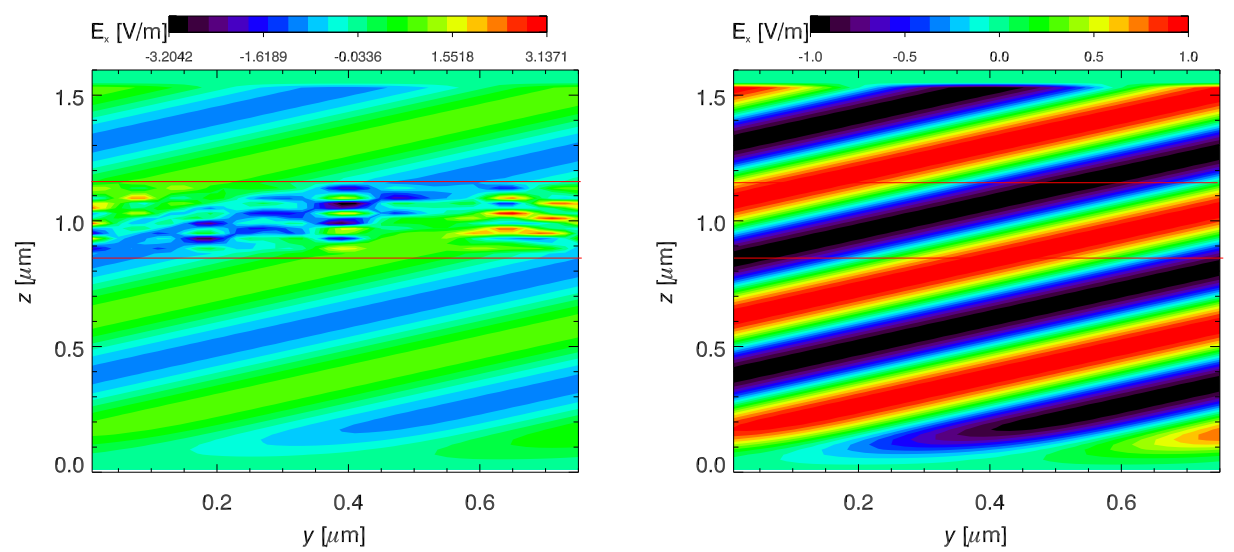

FIG. 5.1. Onset of a trapping instability for the (TM) case using the TMQuLin1Lift algorithm on a single refinement patch after $2 \times 10^{4}$ iterations (left). Stable behavior of the TMQuQuHav algorithm on the same computational domain (right).

of 74.64 degrees. To show this instability the parameters were chosen according to the analytical prediction discussed in the previous section: $\lambda=83.7758 \mathrm{~nm}, k_{y} \Delta y=$ $\pi / 2$ and $\omega \Delta t=0.6$. In Fig. 5.2 we show the solution before and after the onset of the instability, for $5 \times 10^{3}$ and $1.2 \times 10^{4}$ coarse iterations, respectively. The growing oblique wave trapped inside the coarse strip can be clearly seen in this figure. The wavenumber of the trapped wave is the same as the wavenumber of the source, $k_{y} \Delta y=$ $\pi / 2$, corresponding to half of the Nyquist wavenumber along the interface.
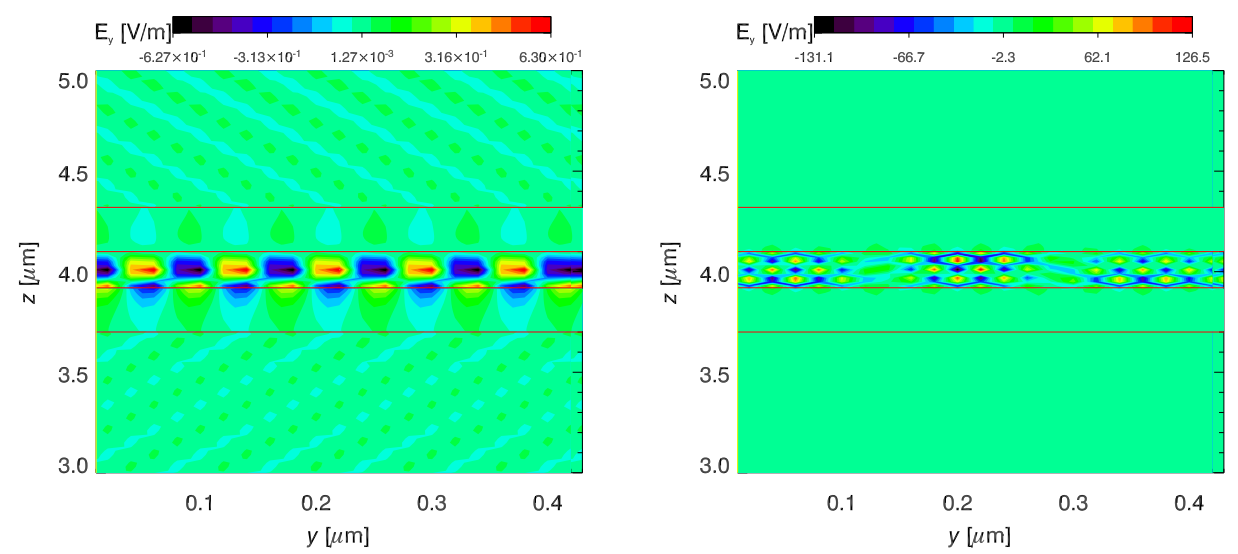

FIG. 5.2. Onset of a trapping instability for the (TE) case using the TEQuLift algorithm on a computational domain consisting of two refinement patches surrounding a coarse strip after $5 \times 10^{3}$ (left) and $1.2 \times 10^{4}$ (right) iterations.

Example 3. (TE) White noise initial data.

In this example we use a white noise initial condition on the whole coarse domain that contains two fine patches as shown in Fig. 5.3. Reflecting boundary conditions $(\mathbf{E}=0)$ were used in all directions. For the TEQuLift algorithm a trapping instability 
formed inside the fine patches and on the coarse grid between the fine patches, shown in the figure after $5 \times 10^{4}$ coarse iterations. The TEQuHav algorithm remained stable after $7.5 \times 10^{4}$ iterations but introduced some damping of the solution due to waves crossing the grid interfaces multiple times upon reflection from the boundaries. Damping of the solution at grid interfaces suggests that for such algorithms AMR gridding should not be used for simulations involving numerous grid interface crossings.
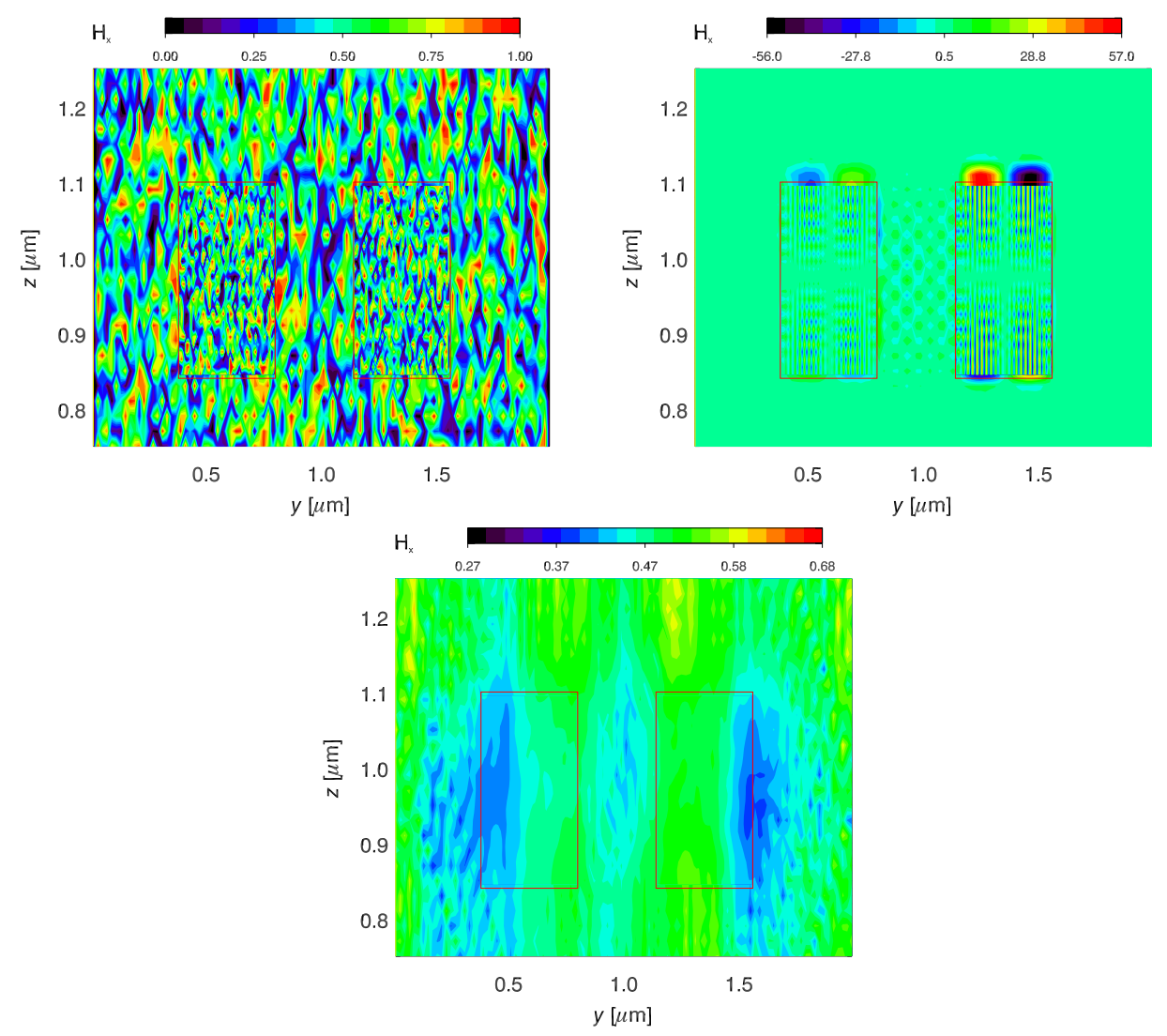

FIG. 5.3. Onset of a trapping instability for the TEQuLift algorithm, for a white noise initial condition (top left), inside the fine patches after $5 \times 10^{4}$ iterations (top right). Stable behavior of the TEQuHav algorithm on the same computational domain (bottom) after about $7.5 \times 10^{4}$ iterations.

\section{Conclusions}

When the neutrally stable Yee algorithm is extended to include adaptive mesh refinement it becomes extremely sensitive to perturbations introduced by the interpolation algorithms at grid interfaces potentially resulting in trapping instabilities, exponentially growing modes, mode resonances with the interface and mode-mode resonances. Gustafsson-Kreiss-Sundstrom-Trefethen analysis based on the computation of the reflection/transmission coefficients and mode analysis of the solution on the infinite domain with a single interface and matrix stability analysis on finite domains was applied to several interpolation methods for 2D AMR-FDTD algorithms. Since these tests are only necessary conditions for stability, the algorithms have to be further tested for possible resonant unstable behavior on a finite domain with perfectly 
reflecting boundaries.

Acknowledgments. Effort sponsored by the Air Force Office for Scientific Research, Air Force Materiel Command, USAF, under grant numbers FA9550-04-1-0213 and F49620-03-1-0194. The work of MB was supported in part by NSF grant ITR0325097.

\section{REFERENCES}

[1] M. J. Berger and J. Oliger, Adaptive mesh refinement for hyperbolic partial differential equations, J. Comput. Phys., 53, 484-512, 1984.

[2] M. J. Berger, Stability of interfaces with mesh refinement, math. Comp., 45, 301-318, 1985.

[3] M. J. Berger and R. J. LeVeque, Adaptive mesh refinement using wave-propagation algorithms for hyperbolic systems, SIAM J. Num. Anal., 35, 2298-2316, 1998.

[4] R. Biedron and J. Thomas, A generalized patched-grid algorithm with application to the F-18 forebody with actuated control strake, Computing Systems in Engineering, 1(2-4), 563-576, 1990.

[5] M. W. Chevalier and R. J. Luebbers, FDTD local grid with material traverse, IEEE Tran. Anten. Prop., 45, 411-421, 1997.

[6] F. Collino, T. Fouquet and P. Joly, A space-time mesh refinement method for the 1D Maxwell's equation, C. R. Acad. Sci. Paris, 328, 263-268, 1999.

[7] F. Collino, T. Fouquet and P. Joly, Conservative space-time mesh refinement methods for the FDTD solution of Maxwell's equations, J. Comput. Phys., 211, 9-35, 2006.

[8] B. Gustafsson, H. O. Kreiss and J. Oliger, Time Dependent Problems and Difference Methods, New York, Wiley, 1995.

[9] B. Gustafsson, H. O. Kreiss and Sundström, Stability theory of difference approximations for mixed initial boundary value problems II, Math. Comp., 26, 649-686, 1972.

[10] R. L. Higdon, Radiation boundary conditions for elastic wave equation, SIAM J. Num. Anal., 27, 831-869, 1990.

[11] R. L. Higdon, Numerical absorbing boundary conditions for wave equation, Math. Comp., 49, 65-90, 1987.

[12] N. J. Higham, P. A. Knight, Matrix powers in finite precision arithmetic, SIAM J. Matrix Anal. and Appl., 16(2), 343-358, 1995.

[13] R. J. LeVeque, O. Steiner and A. Gautschy, Computational Methods For Astrophysical Fluid Flow, Berlin ; New York, Springer, 1998

[14] R. B. Lehoucq, D. C. Sorensen and C. Yang, ARPACK Users' Guide: Solution of Large-Scale Eigenvalue Problems with Implicitly Restarted Arnoldi Methods, SIAM, Philadelphia, PA, 1998.

[15] P. Monk, Sub-gridding FDTD schemes, ASES Journal, 11, 37-46, 1996.

[16] M. Okoniewski, E. Okoniewski and M. A. Stuchly, Three-dimensional subgridding algorithm for FDTD, IEEE Trans. Antennas Prop., 45, 422-429, 1997.

[17] R. F. Remis, On the stability of the finite-difference time-domain method, J. Comput. Phys., 163, 249-261, 2000 .

[18] R. Rieben, D. White and G. Rodrigue, High-order symplectic integration methods for finite element solutions to time dependent Maxwell equations, IEEE Trans. Antennas Prop., 52, 2190-2195, 2004.

[19] R. Schuhmann and T. Weiland, Conservation of discrete energy and related laws in the finite integration technique, Progress in Electromagnetics Research, PIER 32, 301-306, 2001.

[20] A. Taflove and S. C. Hagness, Computational Electrodynamics: the FDTD method, 2nd ed., Boston, London, Arthech House, 2000.

[21] M. Thuné, A numerical algorithm for stability analysis of difference methods for hyperbolic systems, SIAM J. Sci. Stat. Comp., 11, 63-81, 1990.

[22] P. Thoma and T. Weiland, A consistent subgridding scheme for the finite difference time domain method, Int. J. Num. Modeling: Elec. networks, devices and fields, 9, 359-374, 1996.

[23] L. N. Trefethen, Instability of difference models for hyperbolic initialboundary value problems, Comm. Pure and Appl. Math., 37, 329-367, 1984.

[24] L. N. Trefethen, Group velocity interpretation of the stability theory of Gustafsson, Kreiss and Sundström, J. Comput. Phys., 49, 199-217, 1983.

[25] L. N. Trefethen, Stability of finite difference models containing two boundaries or interfaces, Comm. Pure and Appl. Math., 37, 329-367, 1984. 
[26] M. J. White, M. F. Iskander and Z. Huang, Development of multi-grid FDTD code for threedimensional applications, IEEE Trans. Antennas Prop., 45, 1512-1517, 1997.

[27] K. S. Yee, Numerical solutions of initial boundary value problems involving Maxwell's equations in isotropic media, IEEE Tran. Anten. Prop., AP-14, 302-307, 1966.

[28] A. Yefet and P. G. Petropoulos, A staggered fourth-order accurate explicit finite difference scheme for the time-domain Maxwell's equations, J. Comput. Phys., 168, 286-315, 2001.

[29] A. R. Zakharian, M. Brio and J. V. Moloney, FDTD based second-order accurate local mesh refinement method for Maxwell's equations in two space dimensions, Comm. Math. Sci., 2(3), 497-513, 2004.

[30] A. R. Zakharian, M. Brio, C. Dineen and J. V. Moloney, Second-Order Accurate FDTD Space and Time Grid Refinement Method in Three Space Dimensions, Phot. Tech. Lett., to appear, 2006

[31] S. Zhao and G. W. Wei, High-order FDTD methods via derivative matching for Maxwell's equations with material interfaces, J. Comput. Phys., 200, 60-30, 2004. 